\title{
2004 MRS Fall Meeting Reveals Global Impact and Opportunities of Materials Research
}

On the opening day of the 2004 Materials Research Society Fall Meeting, Thomas M. Menino, mayor of the city of Boston, proclaimed Monday, November 29, 2004, to be Materials Science Day. Through the duration of the Meeting, held in Boston, Mass., the MRS science exhibition Strange Matter was on display at the Boston Museum of Science, where it was hosted from October 2004 to January 2005. Mayor Menino's proclamation recognized the science exhibition as well as the $3000-5000$ international attendees and exhibitors MRS has drawn to Boston every fall for 27 years in order to provide a venue for researchers to communicate and network with their peers for the advancement of materials research and improvement of the quality of life.

The Meeting Chairs, Shefford P. Baker (Cornell University), Julia Hsu (Sandia National Laboratories), Bethanie J.H. Stadler (University of Minnesota), and Richard Vaia (Air Force Research Laboratory), weaved their magic as they combined public outreach with the 42 technical symposia, plenary session, and awards ceremony, for the Meeting held November 29-December 3 at the Hynes Convention Center and the Sheraton Boston Hotel and Towers. The MRS President's Reception was held at the Strange Matter exhibition at the Museum of Science on Sunday evening, following a special program at the exhibition that afternoon for high school science teachers. MRSEC celebrated its 10 th anniversary on Tuesday evening at the Fall Meeting venue, where all of the centers offered poster presentations on their research and educational outreach programs. U.S. and international funding seminars were held, delineating opportunities in research and international collaborations. The Meeting concluded after six days of more than 4200 scheduled technical talks, along with poster sessions, tutorials, special events, the equipment exhibit and Research Tools Seminars, and other activities.

\section{Technical Talks}

"Science is People," said Alan MacDiarmid (Univ. of Pennsylvania), 2000 Chemistry Nobel Laureate, speaking at the NSF MRSEC program celebration of its 10th anniversary held at the 2004 MRS Fall Meeting. The work of science is to offer solutions for problems that face humankind. And currently, he said, a cheap alternative source for energy is the solution for a number of those problems.

"We need an international crash research program similar to the Apollo moon program last century," MacDiarmid said. His message on Tuesday evening was the same one espoused by plenary speaker Mildred S. Dresselhaus (MIT) on Monday evening and Symposium X speaker Richard E. Smalley (Rice Univ.), 1996 Chemistry Nobel Laureate. The Meeting attendees agreed as they filled sessions such as Symposium M on Materials Aspects of Fuel Cells within the topic cluster of Energy Generation and Storage.

Internationally, policymakers are trying to turn to science to solve their energy needs as they steer funds toward a "hydrogen economy." Smalley noted that the U.S. president, in his second term, "has a real opportunity to make a difference." In particular, Smalley said the president should inspire the next generation of U.S. scientists and engineers, since so few Americans are pursuing scientific and engineering careers. Placing the global energy crisis at the top of a list of 10 problems facing humanity for the next 50 years, Smalley said that abundant, low-cost clean energy would take care of the remaining problems such as water, food, poverty, and population. Global warming, he said, is also a major issue that the president should address.

Smalley then described possible ways in which the energy problem can be solved. Oil was the basis of energy prosperity for the world in the 20th century. Sometime soon, however, worldwide oil production will level out and then start to decline. Energy prosperity for primary energygeneration in the 21st century is likely to be an array of many technologies such as clean coal, natural gas, nuclear, solar, wind, and biomass. Smalley proposed a world energy scheme-the distributed storage grid-to supply the 30-60 tera-

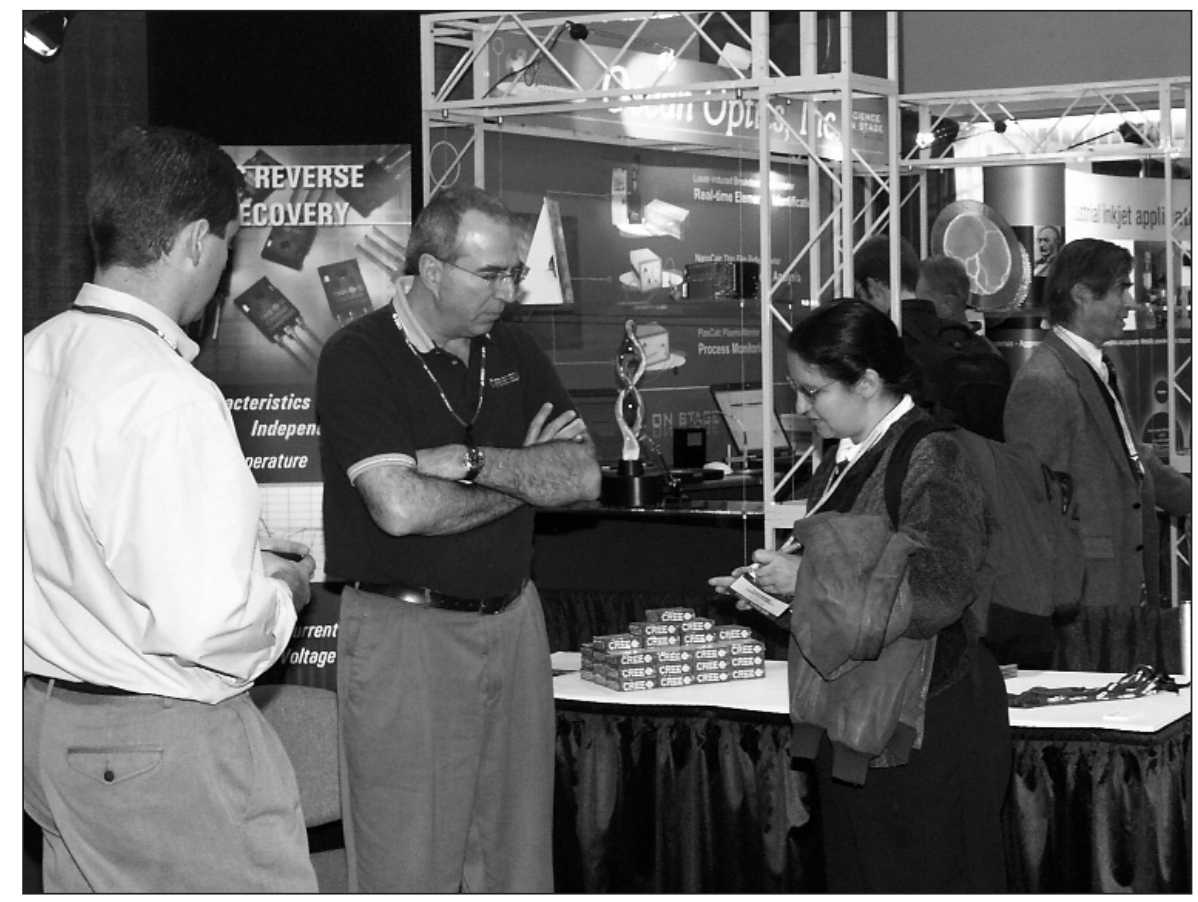

Equipment exhibitors discuss products and services with meeting attendees. 
watts of energy that will be required in 2050. This involves transporting terawatts of power over continental distances by high-voltage transmission lines and local storage of this electrical energy in "uninterruptible power supplies" in every house, business, and vehicle. Smalley said that innovations in nanotechnology and associated materials advances will play a crucial role in developing this electrical grid of the future.

Dresselhaus also pointed to the appeal of nanotechnology in solving future energy needs. In discussing the importance of and the drivers for the hydrogen economy, Dresselhaus discussed the significance of nanostructures. In her broad introduction to the burgeoning field of nanoscience and nanotechnology, she described one-dimensional nanostructures, including nanowires, nanotubes, and atomic lines, and she overviewed the special properties of 1D systems. Dresselhaus turned to the younger scientists in the audience for their critical role in this regard to come up with new and innovative ideas.

In the cluster of symposia on Novel Materials Fabrication and Applications, nanoscience was heavily discussed. In Symposium HH, G. Duesberg (Infineon Technologies, Germany) outlined a few schemes for the integration of carbon nanotubes into microelectronics. Duesberg discussed the lithographically defined vertical growth of MWNTs, which are able to carry current densities of up to $5 \times 10^{8}$ $\mathrm{A} / \mathrm{cm}^{2}$ and can potentially be used as interconnects. Further work at Infineon has been directed at shrinking the lateral dimensions of nanotube devices. Duesberg reported the fabrication of "the world's smallest nanotube field-effect transistor," with a channel length of $<20 \mathrm{~nm}$. These devices have an on/off ratio of up to 106 and are able to switch ultrahigh currents of $>15 \mu \mathrm{A} /$ tube. To overcome the various challenges in nanotube electronics, such as reliability and reproducibility, researchers are working on the vertical carbon nano- tube concept, where vertically grown parallel arrays of nanotubes can be grown controllably between desired heights of the source and drain. Researchers at Infineon have recently developed a nanotube-based power transistor capable of operating with macroscopic loads.

In Symposium II on modeling one- and two-dimensional nanomaterials, S.G. Louie (UC-Berkeley, LBNL) observed that the restricted geometry and symmetry of nanostructures often give rise to interesting quantum confinement, enhanced many-electron interactions, and other effects related to reduced dimensionality. Louie also described the optical spectra of small-diameter carbon nanotubes, which exhibit dramatic excitonic effects in both metal and semiconducting tubes. In related sessions on the electronic and optical properties of 1D systems, A. Rubio (University of the Basque Country, Spain) discussed the theoretical characterization of optical absorption spectra. As Rubio stated, for quantitative predictions, a simple

ACRONYM KEY

1D: one-dimensional

2D: two-dimensional

3D: three-dimensional

AFM: atomic force microscopy

AFRL: Air Force Research Laboratory

AIST: Agency of Industrial Science and

Technology

ANL: Argonne National Laboratory

ANU: Australian National University

ARL: U.S. Army Research Laboratory

ARO: Army Research Office

BAW: bulk acoustic wave

BNL: Brookhaven National Laboratory

CalPoly: California Polytechnic State

University

CalTech: California Institute of Technology

CMR: colossal magnetoresistive

CNRS: Centre National de la Recherche Scientifique

CSIRO: Commonwealth Scientific and

Industrial Research Organisation

CVD: chemical vapor deposition

cw: continuous wave

DOE: U.S. Department of Energy

EPFL: Ecole Polytechnique Fédérale de Lausanne

EU: European Union

FeRAM: ferroelectric random-access memory

FET: field-effect transistor

GE: General Electric

Georgia Tech: Georgia Institute of Technology

GM: General Motors

GMR: giant magnetoresistance

HF: hydrofluoric acid

HP: Hewlett-Packard

HRTEM: high-resolution transmission

electron microscopy
IC: integrated circuit

ICP-MS: inductively coupled plasma-mass spectrometry

IMRE: Institute of Materials Research and

Engineering

IMT: Institut für Mikrostrukturtechnik des Forschungszentrums

IR: infrared

JHU: The Johns Hopkins University

LAAS-CNRS: Laboratory for Analysis and

Architecture of Systems-Centre National de

la Recherche Scientifique

LANL: Los Alamos National Laboratory

LBNL: Lawrence Berkeley National Laboratory

LED: light-emitting diode

LLNL: Lawrence Livermore National Laboratory

LZH: Laser Zentrum Hannover

MBE: molecular-beam epitaxy

MEMS: microelectromechanical systems

MIT: Massachusetts Institute of Technology

MPI: Max Planck Institute

MQW: multiple quantum well

MRAM: magnetic random-access memory

MRSEC: Materials Research Science and

Engineering Center

MTJ: magnetic tunnel junction

MWNT: multiwalled carbon nanotube

NASA: National Aeronautics and Space

Administration

NCSR: National Center of Scientific Research

NCSU: North Carolina State University

NIH: National Institutes of Health

NIST: National Institute of Standards and

Technology

NMR: nuclear magnetic resonance

NRC: National Research Council
NREL: National Renewable Energy Laboratory

NRL: Naval Research Laboratory

NSF: National Science Foundation

OLED: organic light-emitting diode

ONR: Office of Naval Research

ORNL: Oak Ridge National Laboratory

OSU: Ohio State University

PNNL: Pacific Northwest National Laboratory

PSI: Paul Scherrer Institute

PSU: The Pennsylvania State University

PV: photovoltaic

PZT: lead zirconium titanate

R\&D: research and development

RPI: Rensselaer Polytechnic Institute

SAXS: small-angle $x$-ray scattering

SEM: scanning electron microscopy

SNL: Sandia National Laboratories

SOFC: solid-oxide fuel cell

SPM: scanning probe microscopy

STEM: scanning transmission electron

microscopy

SUNY: State University of New York

SWNT: single-walled carbon nanotube

TEM: transmission electron microscopy

UC: University of California

UCLA: University of California-Los Angeles

UCSB: University of California-Santa Barbara

UCSD: University of California-San Diego

UHV: ultrahigh vacuum

UIUC: University of Illinois at Urbana-

Champaign

UST: University of Science and Technology

UV: ultraviolet

VCSEL: vertical-cavity surface-emitting laser

XAS: $x$-ray absorption spectroscopy

XRD: $x$-ray diffraction 
calculation based on the assumption of non-interacting particles and on the electronic band structure on the density functional theory level is not accurate enough; Rubio and co-workers calculated the excitonic effects by the solution of the Bethe-Salpeter equation.

S.C. Glotzer (Univ. of Michigan) presented an overarching view of novel materials based on the functionalization of nano building blocks, including nanospheres, nanorods, nanocubes, nanoplates, and nanoprisms, with biomolecular "handles" and organic tethers, that will provide new possibilities for directing self-assembly of complex structures. Glotzer described a number of examples where molecular simulation can yield insight into the conditions under which various target structures may be achieved. Most recently, molecular simulations were performed to study the self-assembly of particles with discrete attractive interaction sites—"patches" — at prescribed locations on the particle surface. The results showed how precise arrangements of patches combined with patch "recognition" or selectivity may be used to control the relative position of particles and the overall structure of particle assemblies. In this context, patchy particles represent a new class of building blocks for the fabrication of precise structures.

A study on molecular simulation of self-association of modified buckyballs $\left(\mathrm{C}_{60}\right)$ in aqueous solution was presented by G.D. Smith (Univ. of Utah). As Smith described, modification of fullerenes by grafting a low molecular weight watersoluble poly(ethylene oxide) (PEO) can solubilize the buckyballs. By controlling the number of grafted chains, their molecular weights, and how the chains are distributed on the buckyball surface, it is possible to control the interactions between modified buckyballs and induce highly asymmetric interactions between them. As such, interactions could lead to a myriad of self-assembled structures and phases, chemical modification appears to be a promising route to obtaining nanoscale building blocks for novel materials, Smith said. Smith described specific interactions from molecular dynamics simulations. A coarse-grain representation was derived based upon the potential of mean force between modified buckyballs as a function of separation and orientation, including solventinduced interactions and asymmetric interactions due to the distribution of PEO chains. Smith also discussed various structures and phases predicted by the model to be obtainable from a solution of PEO-modified buckyballs.

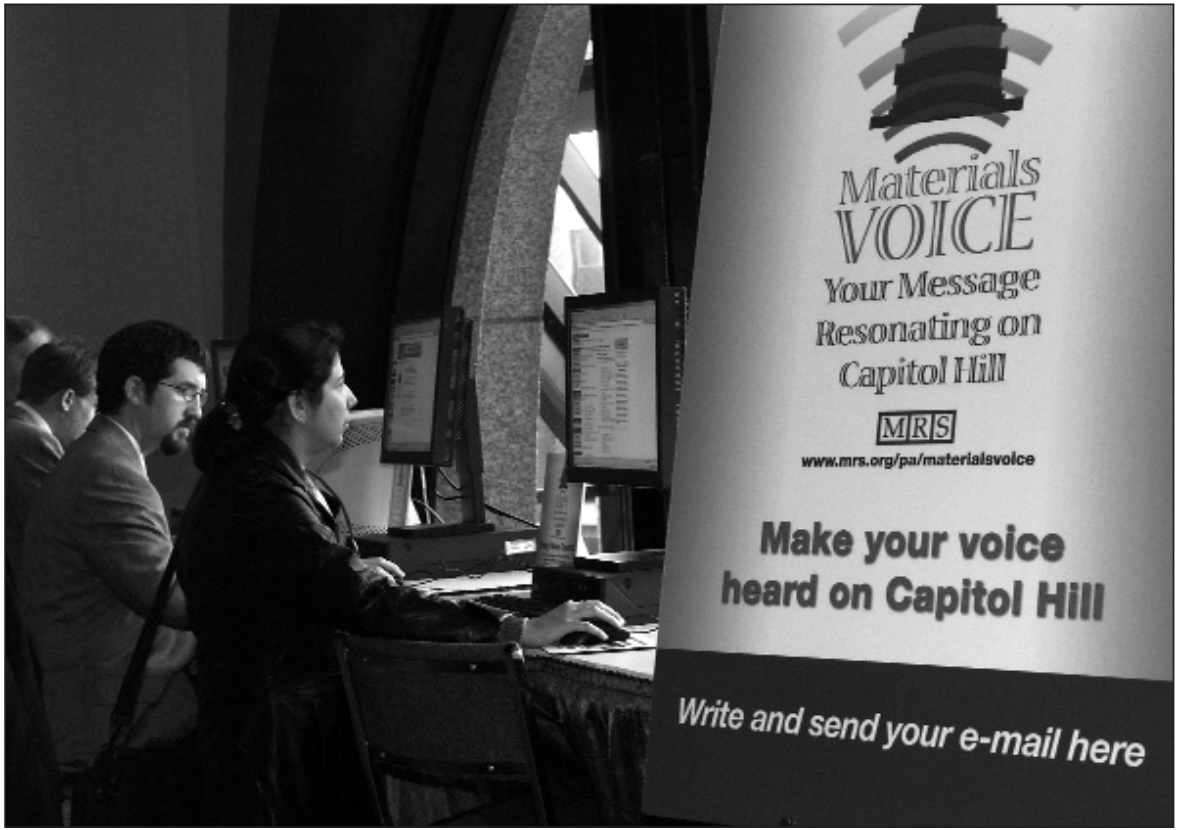

Computer kiosks were set up to enable meeting attendees to send letters to their legislators through MRS Materials Voice, accessed at www.mrs.org/pal.

In Symposium GG, O.D. Velev (NCSU) outlined strategies for assembling microparticles, nanoparticles, and living cells in one or two dimensions using ac dielectrophoresis. Particle chaining forces and positive dielectrophoresis led to the assembly of latex particles in a singlecrystalline domain, with only six firstorder spots in its laser diffraction pattern. Metallic nanoparticles placed in the ac field were driven by the electric-field gradient to form microwires. Velev and his co-workers were able to vary experimental conditions such as frequency and solvent viscosity to control branching in these structures. Velev further demonstrated the use of ac fields to direct the assembly of live yeast cells in composites with latex particles. 1D and 2D assemblies can be made by use of the induced dipoles generated in the cells when placed in an ac field. These cell-particle assemblies are expected to be useful as biosensors, bioelectronic circuits, biocompatible coatings, and in microsurgery.

Diatoms and other organisms are capable of producing exquisitely sculpted silica architectures by using $40 \mathrm{~nm}$ or smaller confined-space vesicles for both synthesis and processing. Matching the complexity of silicon chemistry in nature has proven to be a challenge for material scientists. In Symposium GG, G.D. Stucky (UCSB) gave an overview of his research on how emergent materials, the evolution of entirely new properties with increas- ing physical size, can be generated using molecular assembly. A key question has been to study how to control the nonlinear chemical organization processes that occur in confined spaces. Stucky and his co-workers have recently carried out the growth of composite silica mesostructures in nanoporous anodized alumina membranes, where the alumina pore sizes are systematically varied from $18 \mathrm{~nm}$ to $100 \mathrm{~nm}$. Stucky described the structure-selective synthesis of arrays of organic/silica composite fibers within these membranes. The alumina pore size profoundly affects the composite fiber structure and shapes, such as single and double rows of cages; single-, double-, and triple-helix structures; and concentric donuts, obtained at different pore sizes. The organic component of the silica fibers can be removed and the porous silica backfilled with metals or metal oxides to make mesosculptured nanowire arrays. The predictive design of the composite silica assembly has been demonstrated using self-consistent field modeling of the molecular assembly process.

Y. Xia (Univ. of Washington, Seattle), in Symposium FF, noted that while much research in nanoscale science has focused on the size of nanoparticles, the shape of nanoparticles can also significantly impact their properties. Using gold as an example, he said that due to splitting in the surface plasmon resonance peak on changing the shape of gold nanoparticles, the colors of 


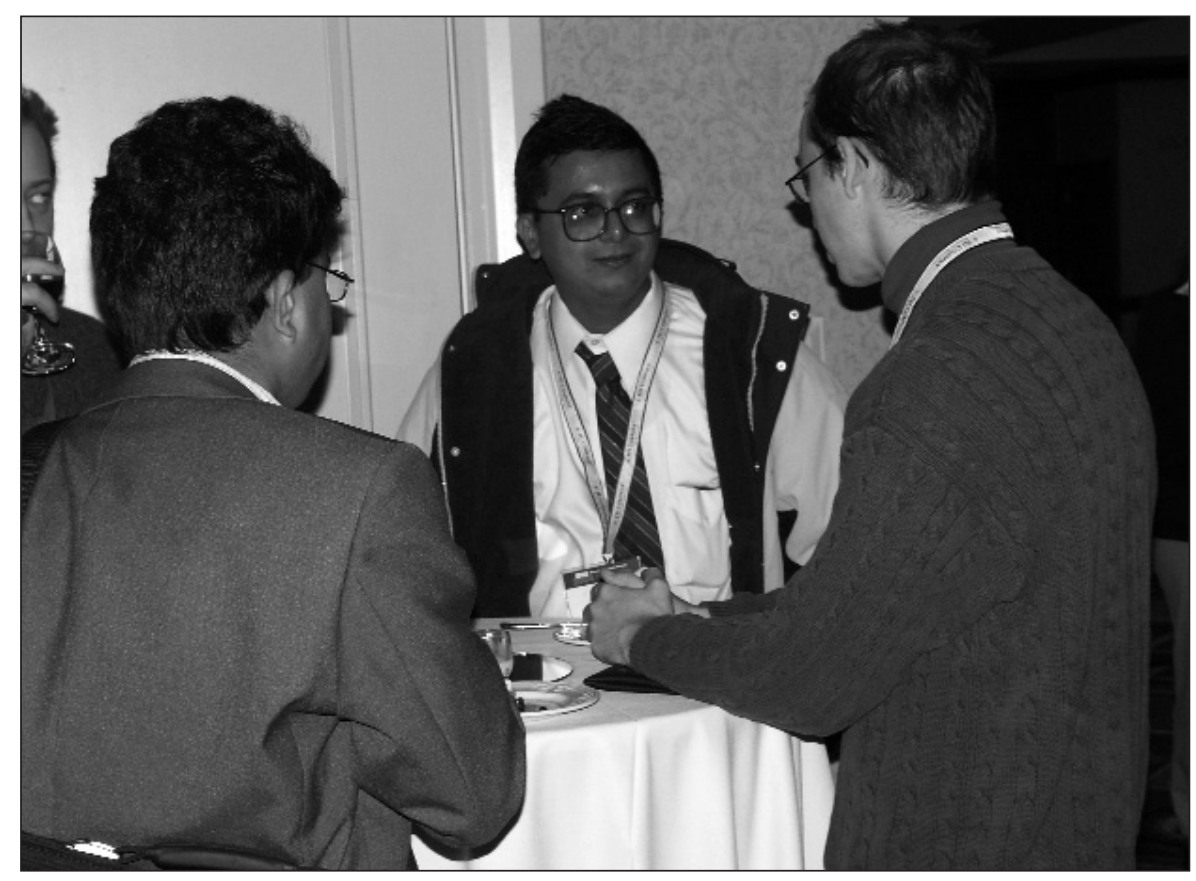

The Student Mixer was held Monday evening.

gold nanoparticles of different shapes can vary significantly. Research in Xia's laboratory focused on the shape-controlled synthesis of metal nanostructures. The use of capping agents that bind selectively to specific facets of growing nanostructures allows the growth of silver nanowires. Xia further demonstrated that controlling the growth kinetics in the polyol reduction of a platinum precursor led to the growth of platinum rods and wires. Recent research in Xia's group focused on the galvanic replacement of gold by silver, resulting in the formation of interesting structures such as Ag/Au MWNTs and "nanorattles."

Rare-earth-doped optical-fiber lasers can provide very high $\mathrm{cw}$ power and have also become attractive as optical pulse amplifiers. However, the high power and small fiber core diameters in these systems can lead to severe nonlinear distortions. One solution, explored by A. Liem's group (Univ. Jena) and reported in Symposium MM, is to use microstructured optical fibers. Tuning the parameters of the microstructure geometry allows single-mode operation while greatly reducing the nonlinear complications. Using such a system, Liem has demonstrated a $260 \mathrm{~W}$ cw laser using a $4 \mathrm{~m}$ fiber with a single transverse mode. The group is pursuing a strategy of replacing all optical elements for pulse generation and control with fiber-based systems that are compact and can be easily integrated.

In Symposium GG, T. Krupenkin (Bell
Labs) outlined recent research on developing dynamically tunable nanostructured surfaces, informally known as "nanograss." The ability to switch local contact angles at liquid-solid interfaces without changing the materials would enable the controllable transport and interaction between a liquid droplet and a solid surface. The use of Si-based nanoposts with a thin layer of dielectric silicon oxide coated with a fluoropolymer leads to the formation of a superhydrophobic surface, where liquid droplets form a rolling ball. Using principles of electrowetting, Krupenkin and his coworkers apply a voltage to these nanoposts, which changes the local contact angle and leads to the droplet penetrating to the solid surface through the nanograss to form a "sticky droplet." Thus, through application of a voltage between the surface and the droplet, the surface can be transformed from superhydrophobic to hydrophilic and even to a surface that is completely wetted by the liquid. Potential applications include "lab-on-achip" technology and reserve nanobatteries, as well as reducing drag in objects used underwater.

The long wait for commercial carbon nanotube-based electronic devices is almost over, according to J.-C. Gabriel from Nanomix Inc., a California-based startup company that expects to begin marketing SWNT-based hydrogen sensors this year. Delivering an invited talk at
Symposium HH, Gabriel discussed the tremendous versatility of sensors based on carbon nanotube FETs, which he compared to nerve cells capable of sensing different stimuli. While single-nanotube transistors are too fragile to be useful over long periods of time, networks and arrays of nanotubes make robust sensors while also giving high signal-to-noise ratios with low power consumption, therefore enabling wireless application. The tremendous sensitivity of the transport properties of SWNT devices to small environmental changes is ideal for sensing applications but also requires that nanotubes be functionalized to be selective and specific for certain analytes. Metal functionalized nanotubes make for excellent hydrogen sensors capable of detecting levels of hydrogen with great sensitivity. Other applications being explored at Nanomix include a polymer functionalized SWNT sensor for $\mathrm{CO}_{2}$ and biofunctionalized nanotube sensors for biomolecule detection in liquid environments.

In Symposium MM, K. Sugioka (RIKEN) reported progress on lab-on-achip device manufacturing. These devices combine microfluidic, micromechanical, and micro-optical components that can be used for field-deployable chemical analysis with high efficiency, high accuracy, and high performance. Such devices can be used for human gene and protein analysis, medical inspection, and new drug development. Sugioka showed how 3D hollow microstructures in photosensitive glass can be made by direct-writing latent images in the glass using a femtosecond laser, baking the samples, and then etching them in diluted HF for selective removal of modified regions. Structures were demonstrated that combine streams of fluids and control their flow with microvalves, which then can be analyzed with light controlled by micromirrors, all on a chip.

Bloch oscillations and Zener tunneling are well-characterized phenomena for electrons in solid-state systems with an electric bias field. In Symposium B, L. Pavesi (Univ. Trento, Italy) reported the first observations of these effects in photonic systems. Using a porous-silicon optical superlattice consisting of 10 coupled microcavities, Pavesi's group imposed an effective bias field for photons in the superlattice by introducing an index of refraction gradient along the cavities-and hence an optical thickness gradient. Optical pulses traveling through these biased superlattices were observed to break into pulse trains whose period depended on the strength of the bias field-the optical analogue of Bloch oscil- 
lations. For a narrow range of bias fields, the group also observed Zener tunneling of photons between adjacent momentum bands, as evidenced by strongly enhanced transmission in optical superlattice samples with the correct gradient. The discoveries may lead to new applications in photonic engineering, including devices for photonic circuits.

In the first session of Symposium J on Magnetic-Photonic Crystals, S. Kahl (Swedish Royal Inst. of Tech.) gave a presentation on the magneto-optical response of a 1D all-garnet photonic crystal in trans- mission and reflection. Kahl's group fabricated and tested a magnetophotonic crystal made of alternating bismuth iron garnet and yttrium iron garnet (BIG and YIG) layers, with a total thickness of $1.5 \mu \mathrm{m}$ and a transmission peak of $\sim 750 \mathrm{~nm}$. The structure contains an extended central BIG layer that acts as a Fabry-Perot cavity and displays high transmission and Faraday rotation. Kahl also studied the system in reflection by coating one side with a Ag reflection layer, which led to strongly enhanced Faraday rotation at the transmission peak. He proposed using reflection-coated mag- netophotonic crystals in magneto-optical imaging, predicting good contrast and sensitivity for such a technique.

In the area of materials characterization, P.M. Voyles (Univ. of Wisconsin), in Symposium $\mathrm{P}$, addressed the issue of how researchers can be sure that they are imaging single atoms when using Z-contrast STEM. It is just a question of the right place, the right number, and the right intensity, he said. The right place means that the image appears where it should, rather than where it should not. The right number means that the number of impuri-

\section{Poster Prizes Awarded at the 2004 MRS Fall Meeting}

The 2004 MRS Fall Meeting Chairs awarded prizes for the best poster presentations. Prize recipients received $\$ 500$, a certificate, and the honor of having their posters on display for the remainder of the meeting. Posters and authors awarded prizes were (A5.13) Preparation of $\mathrm{TiO}_{2}$ Micro Wire Sensor Prepared by Phase Separation-Selective Leaching Method, A. Yasumori, J. Yoshida, H. Matsumoto, and K. Nishio (Tokyo University of Science); (B9.5) Spectroscopic Ellipsometry Study of CdSe and CdTe Nanoparticles Embedded in $\mathrm{SiO}_{2}$ Films, P.B. Dayal, M.B. Raj, and P.D. Paulson (Indian Institute of Technology, Delhi; University of Delaware); (C5.5) Metal Nanotube Membranes with Sub-100-nm Apertures and Their Lithographic Applications, W. Lee, H. Fan, S.-K. Lee, S. Richter, S. Matthias, W. Wulfhekel, M. Zacharias, D. Hesse, J. Kirschner, K. Nielsch, and U. Goesele (Max Planck Institute of Microstructure Physics, Halle), and E. Moyen and M. Hanbuecken (CRMCN-CNRS); (D6.18) A New Crystalline to Crystalline Phase Change Memory Cell Using $\left(\mathrm{Ge}_{1} \mathrm{Sb}_{2} \mathrm{Te}_{4}\right)_{0.8}\left(\mathrm{Sn}_{1} \mathrm{Bi}_{2} \mathrm{Te}_{4}\right)_{0.2}$ Alloy, D.-H. Ahn, H.S. Kwon, M.H. Kwon, H.M. Kim, T.Y. Lee, and K.B. Kim (Seoul National University), and D.H. Kang, T.G. Kim, K.S. Lee, T.S. Lee, I.H. Kim, W.M. Kim, and B. Cheong (Korea Institute of Science and Technology); (E11.10) Crystallinity and Polarity of III-V Nitride Semiconductors Grown on ZnO, T. Ohgaki, N. Ohashi, and I. Sakaguchi (National Institute for Materials Science, Tsukuba), S. Sugimura (Tokyo Denpa Co.; National Institute for Materials Science, Tsukuba), K. Maeda (Tokyo Denpa Co.), M. Sato (Tokyo Denpa Co.; NIMSWave Inc., Gunma), and H. Haneda (National Institute for Materials Science, Tsukuba; Kyushu University, Fukuoka); (H11.13) Magnetic and Structural Characterization of Magneto-Electric Epitaxial Oxide Multilayers, R.V. Chopdekar (Cornell University, University of California-Berkeley) and Y. Suzuki (University of California-Berkeley); (I4.18) Production and Characterization of Ferromagnetic Alloyed Nanowires inside Carbon Nanotubes, A.L. Elias A., J.A. Rodriguez Manzo, A. Zamudio, S. Baltazar Rojas, F. Lopez Urias, E. Munoz Sandoval, and H. Terrones (IPICyT, Mexico), M. Terrones (IPICyT, National Institute for Materials Science, Tsukuba), M. McCartney and D.J. Smith (Arizona State University), and D. Golberg, C. Tang, and Y. Bando (National Institute for Materials Science, Tsukuba); (M5.14) Analytical TEM Study of the Degradation Phenomena in Proton Exchange Membrane Fuel Cell, T. Akita, J. Maekawa, A. Taniguchi, K. Tanaka, M. Kohyama, and K. Yasuda (National Institute of Advanced Industrial Science and Technology, Osaka); (O10.13) Mapping of Local Electronic Properties and Spatially Resolved Magnetoresistance of Nanostructured CMR Thin Films by Scanning Tunneling Microscope, S. Kar

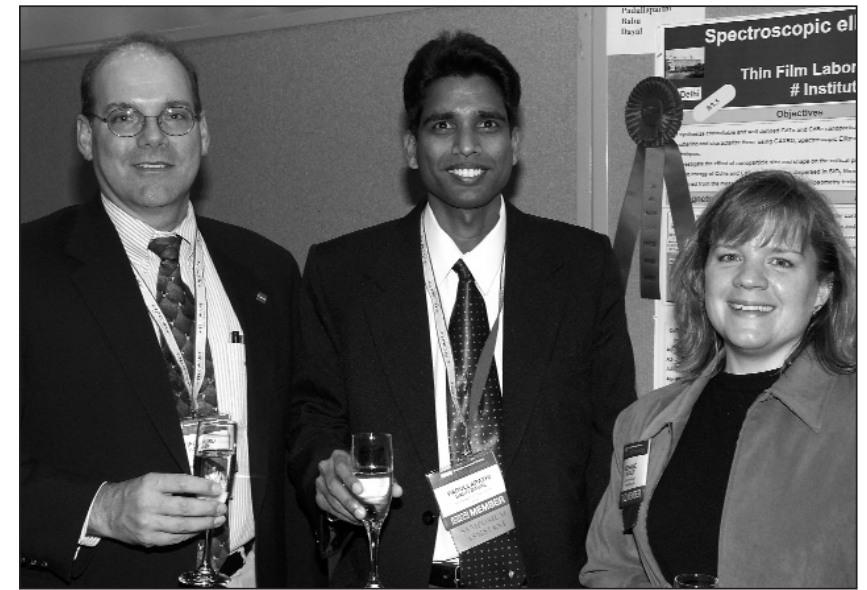

and L.K. Brar (Indian Institute of Science, Bangalore), B. Ghosh and A.K. Raychaudhuri (Indian Institute of Science, Bangalore; National Centre for Basic Sciences, Kolkata), and M.A. Paranjape (Boston College); (P3.3) Electron Holographic Characterization of Nano-Hetero Interface Effect in Gold Catalysts, S. Ichikawa, T. Akita, K. Okazaki, K. Tanaka, and M. Kohyama (National Institute of Advanced Industrial Science and Technology, Ikeda); (R9.9) Experimental and Numerical Analysis of the Interaction between Surface Roughness, Capillarity, and Adhesion in MEMS, F.W. DelRio (Sandia National Laboratories, University of Colorado), M.P. de Boer (Sandia National Laboratories), and M.L. Dunn (University of Colorado); (U11.1) Deformability of Thin Metal Films on Polymer Substrates, T. Li, Z. Huang, and Z. Suo (Harvard University), and S.P. Lacour and S. Wagner (Princeton University); (Y6.14) Time-Dependent Mechanical Behavior of Human Stratum Corneum, K.S. Wu and R.H. Dauskardt (Stanford), E. Bedford (Unilever Research and Development, U.K.), and D.J. Moore (Unilever Research and Development); (FF3.1) Growth of Single-Crystal $\alpha-\mathrm{Fe}_{2} \mathrm{O}_{3}$ from a $\mathrm{CaFe}_{4} \mathrm{O}_{7}$-Based Solvent, A.N. Chiaramonti, J.D. Pless, L. Liu, J.P. Smit, C.H. Lanier, K.R. Poeppelmeier, P.C. Stair, and L.D. Marks (Northwestern); (GG10.20) Actively Controlled Self-Assembly of Colloidal Crystals by Electrocapillary Effect, C.-W. Kuo, J.-Y. Shiu, and P. Chen (Academia Sinica, Taipei); and (KK3.4) Studies of Si Surface Morphology Evolution during $\mathrm{Ar}^{+}$Ion Bombardment, G. Ozaydin, A.S. Ozcan, Y. Wang, J. Hotchkiss, and K.F. Ludwig (Boston University), R.L. Headrick and H. Zhou (University of Vermont), and C.R Eddy (Naval Research Laboratory). 


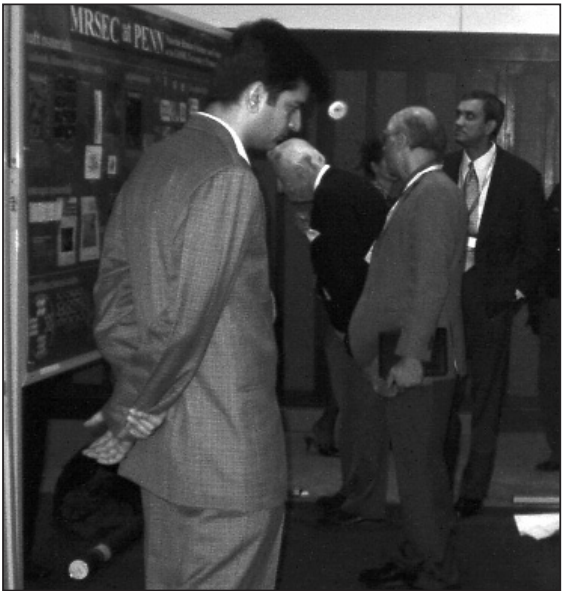

In celebration of its 10th anniversary of the MRSEC program, NSF offered poster presentations on the centers' research and educational outreach programs.

ty atoms is consistent with what is found by other techniques. And the right intensity means that the intensity is aligned with a reasonable model. While it has been possible to image single atoms on a surface since the beginning of STEM use, this work shows how impurities buried in a material can be imaged, determining, for instance, that typically what appears to be 3-4-atom clusters is almost always atoms separated by a thickness of the material.

In the group of symposia on Hybrids and Soft Matter, A. Fuller (Univ. of Florida), in Symposium DD, reported that a wide range of explosive materials display a sharp photoluminescence spectral peak at $705 \mathrm{~nm}$ when they are illuminated with various types of laser light or with xenon lamps. The peak occurs in TNT, nitroglycerin, and other common explosives, but does not appear in related volatile but non-explosive materials such as fertilizer. Fuller's group speculates that the $\mathrm{NO}_{2}$ functional nitro groups present in most explosives may be responsible for the photoluminescence. The discovery could have important applications for detecting explosives at airports, sea ports, and government and military sites.

A newly emerging research area uses bridged aromatic and alkylene silsesquioxanes originally developed by D.A. Loy (LANL), K.J. Shea (UC-Irvine), and J. Moreau (Univ. Montpellier) to create novel hybrid structures. In Symposium EE, S. Inagaki of Toyota Research Laboratories in Japan described the creation of hybrid mesoporous structures using $(\mathrm{RO})_{3} \mathrm{Si}$-aromatic-Si $(\mathrm{OR})_{3}$ systems and surfactant templates. Moreau described the fabrication of chiral rope polysilsesquioxane structures by introducing hydrogen-bonded, self-assembling, chiral (RO) ${ }_{3} \mathrm{Si}-\mathrm{R}-\mathrm{Si}(\mathrm{OR})_{3}$ starting materials. F. Schueth (MPI, Mulheim) discussed the creation of mesoporous catalysts including ones containing paramagnetic particles that allow for separation by magnetic fields.

In Symposium CC, N. Tabiryan said that his group at Beam Engineering for Advanced Measurements Co. in Florida accidentally encountered an extremely strong process for nonlinear absorption in liquid-crystal (LC) materials doped by a dye at a saturating level of concentration. This results in the disappearance of a laser beam with power as high as $20 \mathrm{~mW}$ or more in the material with no visible output. This was observed for a $633 \mathrm{~nm} \mathrm{He}-$ Ne laser beam focused on 30-mm-thick planarly oriented nematic LC (NLC) 5-CB containing up to $2 \mathrm{wt} \%$ of the dye 1,8dihydroxy,4,5-diamino,2,7-diisopentylanthraquinone. The effect can be observed also for a $532 \mathrm{~nm}$ green laser beam in the same material. This "black hole" effect takes place for radiation of sufficiently high power at certain positions of the cell, symmetrically arranged with respect to the focus of a lens (20 mm focal length). Choosing special dyes and solutions may reduce the operation power level and increase the initial transmission state of these materials systems, making them promising for a variety of applications.

In the biophotonics area, A. Fontecchio (Drexel) reported an eye-pressure sensor using holographic polymer-dispersed liquid crystal (HPDLC). The reflectance of a HPDLC film is wavelength-dependent. As the eye pressure changes, the reflected wavelength of the HPDLC changes. By measuring the wavelength shift, the eye pressure can be monitored. In another presentation, N. Lockwood (Univ. of Wisconsin) reported an interesting observation about the active mammalian cells in LC solvent. The well-known 5-CB and E7 LCs are found to be more toxic to mammalian cells than fluorinated biphenyl and terphenyl LC materials. Some cholesterics are also a good solvent for the active cells.

Only $2.5 \%$ of the world's water is fresh water, and only $0.5 \%$ is accessible, while human demands for clean water continue to grow. This is one motivation for the work presented in Symposium BB by A. Mayes (MIT) on self-organized polymer nanofiltration membranes with subnanometer selectivity. Depending on the size of their holes, membranes can be used to filter out impurities, but removing the smallest pathogens can be a daunting task. Membranes are commonly hydrophobic and can easily be fouled by oil and other debris, leading to the need for (and cost of) defouling or replacement. Also, it can be difficult to obtain a high density of pores with a narrow size distribution, which further reduces the efficiency. Mayes presented a way to make polymers such that the backbone is a hydrophobic material but the side chains are hydrophilic, using a poly(vinylidene fluoride) (PVDF) backbone and PEO methacrylate side chains. These materials molecularly self-assemble into a semicrystalline, bicontinuous nanophase domain of PVDF (which provides structural integrity) and PEO (which provides selective transport channels of a well-defined size). The result is reduced fouling and increased absolute flux.

In Symposium Y on the Mechanical Properties of Bio-Inspired and Biological Materials, M. Dao (MIT) described two approaches to modeling cell membranes in order to describe the behavior of red blood cells when they undergo severe elastic deformation. The first model is based on using a continuum concept that includes the shear modulus $\mu$ and bending stiffness $B$, also referred to as the hyperelastic membrane model. The second model starts at the spectrin (protein) level and assumes a worm-like chain model. Dao's group compared the computational results with experiments and found that strain was not distributed uniformly. A solid modeling framework for studying single-cell mechanics and biomechanics, as well as links to diseases, is currently being developed.

In his coverage of the synthesis of bioinspired polymer materials in Symposium Z, M. Kumar (Genencor) discussed a new class of repeat-sequence protein polymers (RSPPs) constituting a new technology with multiple commercial applications based on recombinant DNA technology and fermentation. This technique allows the precise control of the molecular structure and distribution of functional groups in biopolymers to create complex functional biopolymer composites. Putting their research into a historical perspective, Kumar mentioned that researchers have learned to build various devices and machines that are partly inspired by nature and thus have cellular analogues (e.g., collagen for cables and membrane proteins for pumps). However, researchers have not yet achieved the understanding and manufacturing techniques of some of nature's best technologies, such as frictionless electrostatic motors or molecular manufacturing systems. This may represent huge opportunities for research in the coming decades, he said. RSPPs are 


\section{MRS Awards Recognize Scientific and Volunteer Contributions}

The many activities offered by the Materials Research Society are created and carried out largely by the membership. During the plenary session on Monday evening, Merrilea J. Mayo (U.S. National Academies), the 2003 MRS President, acknowledged

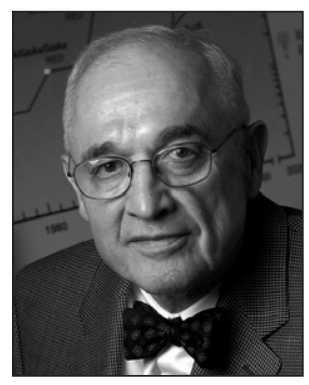

Robert Hull (Univ. of Virginia) and Ted Besmann (ORNL) for their work relating to public policy activities for the Society. Mayo herself was then given a special award for creating and furthering the concept of the Society's Strange Matter science exhibition and personally undertaking fundraising activities essential to bringing the exhibition to life. Alan I. Taub (GM), Alan J. Hurd (LANL), and Shenda M. Baker (Harvey Mudd College) also received special recognition awards for their significant contriNick Holonyak Jr.

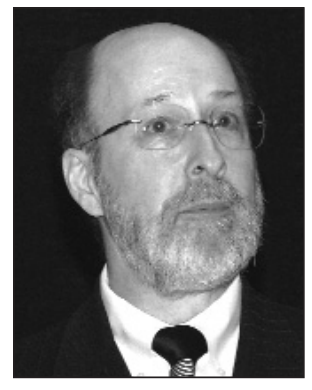
butions to the Strange Matter project.

During the awards ceremony on Wednesday evening, MRS presented its 2004 awards for scientific achievements. The MRS highest honor, the Von Hippel Award, went to Nick Holonyak Jr. (UIUC) for his many contributions to the field of semiconductors. Holonyak was unable to attend the Meeting, so his lecture, "From Transistor to Laser and Light-Emitting Diode," was presented by Russell Dupuis (Georgia Tech). Dupuis began Russell Dupuis presented Nick Holonyak Jr.'s Von Hippel Award lecture at the 2004 MRS Fall Meeting. with a brief video of Holonyak giving a presentation and talking about his mentor, John Bardeen, the only twotime Nobel Laureate in physics. Holonyak was Bardeen's first graduate student. With the discovery of the transistor by Bardeen and Brattain on December 16, 1947-and as a consequence, carrier injection and collection-the hole has indeed become equal to the electron. The semiconductor took on new importance, as did the study of electron-hole recombination, first in the transistor materials $\mathrm{Ge}$ and $\mathrm{Si}$, and then in III-V crystals (e.g., GaAs and GaP). Beyond $\mathrm{Si}$ and its indirect-gap and heterojunction limitations, the directgap III-V materials, particularly III-V alloys, made possible lasers and LEDs, and thus optoelectronics.

The David Turnbull Lectureship was given to Frank S. Bates (Univ. of Minnesota). Bates presented his lecture earlier that afternoon on "Network Phases in Block Copolymer Melts." Bates described an experimental strategy to identify molecular parameters leading to the formation of triply periodic and triply continuous morphologies, referred to as network phases, in copolymers. These intricate structures offer unprecedented opportunities to combine three different polymers, endowed with various physical properties, in single-component selfassembling multicontinuous nanocomposites. Bates described model poly(isoprene- $b$-styrene- $b$-ethylene oxide) (ISO) triblock copolymers that were prepared by anionic polymerization. Small-angle $x$-ray scattering (SAXS) was employed to establish the ordered-state symmetry of these materials. Three different network phases were identified, one with orthorhombic symmetry, flanked as a function of composition by two different cubic phases. Transmission electron microscopy (TEM) resulted in a definitive phase portrait, in addition to dynamic mechanical spectroscopy and birefringence measurements. Recent self-consistent mean-field calculations also provide a fundamental theoretical basis for understanding these results.

MRS Medalists Toh-Ming Lu (RPI) and Sunil Sinha (UC-San Diego), each recognized for their contributions to understanding mechanisms of thin-film surface and interface morphology evolution, gave presentations in the afternoon. In his talk on "Mechanisms on the Morphology of Thin-Film Growth," Lu said that it is well known that during either deposition or etching of a film, the surface morphology often tends to become rough under a variety of processing conditions at sufficiently low substrate temperature and far from equilibrium. Surface morphology controls many physical properties of a film, such as wetting, friction, magnetization, and electrical conductivity. With recent developments in modern characterization tools, including real-space imaging and diffraction techniques, the evolution of growth-front roughness can be studied quantitatively.

$\mathrm{Lu}$ showed that in many growth processes such as sputter deposition and chemical vapor deposition, the experimentally measured growth exponent yields a wide range of values that cannot be explained by existing classes of universality models. The wide range of growth exponent values suggests the existence of diverse roughness possibilities. It is believed that these rich possibilities are due to the competition between the shadowing effect and re-emission effect. Lu argued that shadowing and reemission compete during growth and give rise to a growth exponent depending on the value of the sticking coefficient. Shadowing tends to roughen the surface and re-emission tends to smoothen it. A dynamical equation can be constructed to include both shadowing and re-emission effects.

Sinha's presentation took a different angle, focusing on x-ray and neutron scattering to reveal the nature of surfaces. He used specular reflectivity in which laterally average density profiles normal to the surface are measured to understand surface roughness. In addition to structural roughness, he examined magnetic roughness, essentially the orientation of magnetic domains. These two properties do not necessarily map to each other, so both interfaces need to be considered, and separate structural and magnetic roughness terms can be introduced. Sinha covered recent work related to exchange-bias phenomena (in which the hysteresis curve is shifted from zero). Interfacial spin structure is a key to understanding this exchange bias. Sinha described how measuring reflectivity using resonance $x$-ray scattering can be used as a sensitive technique to determine quantitatively the depth-dependent magnetic density.

Jacob N. Israelachvili (UCSB) received the MRS Medal for work on adhesion and friction. In his talk, Israelachvili reviewed some recent experimental results, including theoretical modeling and computer simulations, on the effects of surface texture, surface energy, and bulk properties on adhesion and friction in materials and, in turn, on some of the fundamental differences between Mode I and Mode II failure of materials. Examples and comparisons included surfaces that are rough or smooth, hard or soft (e.g., viscoelastic), adhesive or non-adhesive, and dry (unlubricated) or lubricated. Recent studies are clarifying the molecular and atomic bases of many well-established adhesion and tribological laws and empirical observations and revealing new insights and relationships between nanoscale (molecular) and macroscale processes. The talk also focused on the sometimes crucial importance of the effects that occur at the sub-nanoscale, that is, in the sub-angstrom or picoscale regime. Israelachvili argued that the ultrafine picoscale details of a surface lattice or its roughness can be the most important factor in determining its friction and Mode II fracture, whereas such effects are quantitatively less important for adhesion and Mode I fracture processes. 
an example of such new technologies, Kumar said. RSPPs are biomaterials formulated to create unique rDNA polymers composed of repetitive protein sequences, such as the silk-elastin family. These bio-inspired RSPPs can find numerous applications in technical textiles and for personal care.

To measure diffusion in metals at low temperatures, "you need a grad student to be around for a few hundred years," said Alex King (Purdue) in Symposium OO on Materials Issues in Art and Archaeology. Since that is not always possible, the usual alternative is to extrapolate from hightemperature behavior. However, such extrapolation is vulnerable to error if the diffusion mechanism changes within the range of the extrapolation. As an alternative, King explored whether an examination of an artifact can shed light on lowtemperature diffusion. King described the examination of a Sheffield plate-fabricated by means of a silver-clad copper manu- facturing technique used between 1740 and 1840 - to understand low-temperature diffusion in the $\mathrm{Cu}-\mathrm{Ag}$ eutectic system. The bond is formed in $1 \mathrm{~min}$ at $780^{\circ} \mathrm{C}$; however, samples have sat for about 200 years at $25^{\circ} \mathrm{C}$. Bulk diffusion profiles can be observed, due to this initial processing, and modeled as the starting point of the longterm, low-temperature diffusion experiment. The samples show the results of grain-boundary diffusion over a few hundred years' time. Comparing the results of these diffusion profiles showed that the diffusion is either the same or lower than that extrapolated by several models, which is good for ensuring stability over long time scales at low temperatures.

Venetian painters of the 1500 s, such as Titian and Giovanni, are noted for the brilliance of their colors, which distinguishes their work from other painters of the day. The discovery of a 1543 materials inventory of a Venetian pigment store has led B. Berrie (National Gallery of Art)

\section{Israelachvili, Lu, and Sinha Receive 2004 MRS Medals}

The Materials Research Society selected three scientists to receive the MRS Medals for 2004, which recognize a specific outstanding recent discovery or advancement that has a major impact on the progress of a materials-related field. Toh-Ming Lu (Rensselaer Polytechnic Institute), and Sunil Sinha (University of California, San Diego/Los Alamos National Laboratory) were each recognized for "seminal contributions to understanding mechanisms of thin-film surface and

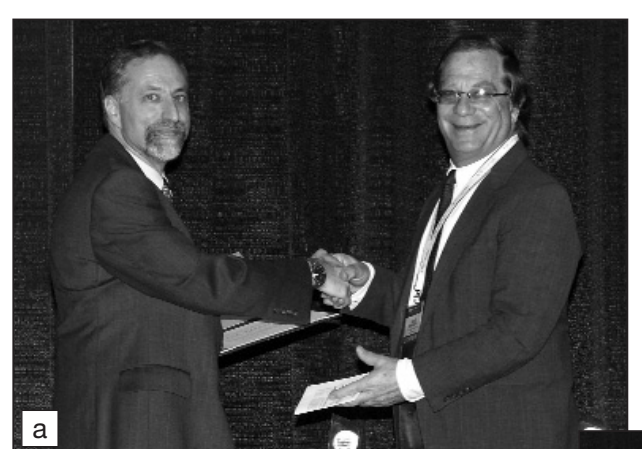
interface morphology evolution and establishing the foundations of diffraction and scattering methods for its quantitative analysis." Jacob Israelachvili (University of California, Santa Barbara) was recognized for "work on adhesion and friction, which has revolutionized the understanding of molecular mechanisms responsible for these technologically vital phenomena."
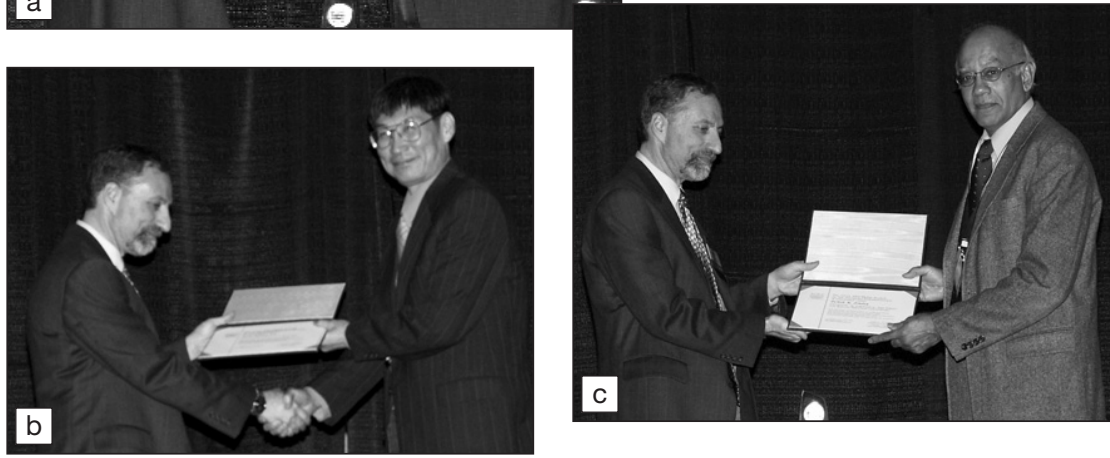

MRS President Howard E. Katz (JHU) presents 2004 MRS Medals to (a) Jacob Israelachvili (UCSB), (b) Toh-Ming Lu (RPI), and (c) Sunil Sinha (UCSD, LANL).

to re-examine the materials that make up the Renaissance Venetian palette. Using SEM and energy-dispersive spectrometry techniques, Berrie found evidence of the use of fine glass particles as paint extenders and colorizers in the works of several Italian painters of the period, including Lorenzo Lotto, Raphael, and Tintoretto. An audience member noted that the optical properties of silicon particles depend strongly on their size and asked whether these painters may have experimented with creating some form of glass-particle light traps in their paints to achieve their brilliant colors, to which Berrie replied that Renaissance painters may have done so, and were likely "better technologists" than is commonly thought.

Also in Symposium OO, S. Miller (NASA Glenn Research Center) discussed atomic oxygen treatment and its effects on a variety of art media. While atomic oxygen treatment was initially developed to study the durability of materials for low-Earth orbit, it has become a useful tool in cleaning works of art. Using a plasma of atomic oxygen, Miller removed soot damage from a variety of art media, including oil and acrylic paint and watercolors, with minimal spectral or brightness change in the materials. The technique can also eliminate a large variety of ink-based graffiti marks, she said. Among various works of art to which it has been applied, Miller's atomic oxygen technique successfully removed a lipstick stain from the valuable Andy Warhol sketch "Bathtub," kissed by an admirer during a 1997 party thrown by a cosmetics company.

In an evening session in Symposium NN on magnetic materials for space applications, K. McNamara talked about the NASA Genesis mission that was launched to collect samples of the solar wind and return them to Earth. McNamara was in charge of recovery of the Genesis spacecraft. Unfortunately, as is well known, the spacecraft crashed to Earth on September 8, 2004, when a parachute failed to deploy. Fortunately, most of the scientific canister and the collectors within were recovered. McNamara said that there are well over 10,000 individually documented collector pieces, all of which are or will be available to scientists for study. She invited interested researchers to contact NASA. McNamara also said that all of the pieces will eventually be fully cataloged in a database that will be made publicly available.

For further details on the technical content of the Meeting, see the following symposium summaries. Proceedings are available on-line at www.mrs.org. 


\section{Semiconductor Sensors Examined (See MRS Proceedings Volume 828)}

The overall aim of Symposium A on Semiconductor Materials for Sensing was to bring together the sensor community to discuss development of new or improved semiconductor materials and the fundamental understanding of the physical/ chemical/biological phenomena at the origin of the sensing mechanism. Several talks covered the highlights of chemical sensors and FET devices, magnetic sensors, radiation sensors, acoustic sensors, mechanical sensors, and biosensors.

T. Kamins (HP) talked about semiconductor sensors that can be integrated onto silicon-integrated circuits and sensors fabricated using IC-based technology. The use of new materials such as metal-catalyzed nanowires and nanotubes was discussed, and the tradeoff between doping and physical size for field-effect devices was considered. Different kinds of sensors detecting various analytes, such as dangerous chemical and biological species, gases, strain, and visible and other radiation, were analyzed, and the characteristics critical for practical applications were reviewed.

J. Schwank (Univ. of Michigan) reviewed the recent advances in combinatorial materials synthesis, theoretical modeling, and simulation of gas-solid interactions based on density functional theory. A combined application of sophisticated experimental and theoretical tools aimed at the design and synthesis of novel sensors might have a lasting impact on general research approaches in the chemical sensor community.

M. Meyyappan (NASA-Ames), V. Dravid (Northwestern), and M.C. Carotta (Univ. Ferrara, Italy) discussed the novelties of 1D and 2D nanostructures for sensing applications. A joint session was held with Symposium K on Solid-State Ionics, addressing solid electrolytes for membrane applications to develop selective sensors.

Symposium Support: APEX Biotechnology Co. and Riken Keiki Co. Ltd.

\section{Semiconductor Research Applied to Terahertz Lasers and Beyond (See MRS Proceedings Volume 829)}

Sandwiched between the domains of electronics and photonics, the terahertz $(\mathrm{THz})$ regime of the electromagnetic spectrum has traditionally lacked mature technologies for generation, mixing, and detection. Yet $\mathrm{THz}$ has important applications, from the detection of chemical and biological agents to imaging and telecommunications uses. Addressing this gap, J. Reno (SNL) presented results from his group in developing $\mathrm{THz}$ quantum cascade lasers in Symposium B on Progress in Semiconductor Materials IV: Electronic and Optoelectronic Applications. Using a resonant tunneling longitudinal optical-phonon scattering technique for lower-level depopulation and a novel double-sided metal-metal waveguide for transverse confinement, Reno's quantum cascade lasers have achieved lasing at milliwatt powers as low as $2.1 \mathrm{THz}(141$ $\mu \mathrm{m})$ at a pulsed operating temperature of $137 \mathrm{~K}$. His group is currently working to reduce the lasing threshold currents and increase the operating range toward room temperature.

In recent years, dramatic success has been seen in the development of semiconductor materials and related quantum structures, including self-assembled quantum dots, for optoelectronic devices such as terahertz devices along with mid-infrared lasers, long-wavelength infrared detectors, modulators, and optical switches. Central to bandgap engineering or, more precisely, "wave-function engineering," is that by spatially varying the composition and doping of semiconductors over distances ranging from a few micrometers down to one monolayer, researchers can tailor the electronic band structure in a nearly controllable and continuous way using epitaxial growth techniques.

VCSELs are promising sources for telecommunications wavelengths, but they generally display undesirable multiple transverse modes. K. Choquette's group (UIUC) demonstrated a singletransverse-mode quantum-well VCSEL with more than $3 \mathrm{~mW}$ of power whose transverse confinement is due to a periodic array of holes etched into the VCSEL surface. The holes form a 2D photonic crystal that supports only one transverse mode when a single hole position is left unetched (a "defect"). The group also demonstrated multilobed far-field laser patterns when several defects are present. The physics of the confinement is the same as that of photonic crystal fibers, although the scaled depth of the holes is much shorter than in the fibers and therefore does not provide as strong a transverse confinement.

Control of the structural properties of quantum dots has seen significant improvements (Y.C. Chua, Univ. of Arkansas; D. Wasserman, Princeton; and C. Jagadish, ANU), resulting in very good optical properties. Also, for quantum wire systems, significant success has been obtained in the control of the structural and optical properties (H. Akiyama, Univ. of Tokyo). Infrared detectors have also improved through advanced growth techniques (F. Szmulowicz, AFRL-WrightPatterson) and by novel detector concepts (M.C. Wanke, SNL).

For electronic devices, advances in materials growth (diamond, III nitrides) were reported. Exciting results on structural properties have been obtained for $\mathrm{ZnO}$ nanowires (D.P. Norton, Univ. of Florida) and UV-emitting ZnO lightemitting devices (D.C. Look, Wright State Univ.). A large potential for applications was reported for $\mathrm{ZnO} / \mathrm{GaN}$ heteroepitaxy (T. Yao, Tohoku Univ.). New insights into the intrinsic optical properties of carbon nanotubes were obtained using magnetospectroscopy (J. Kono, Rice). For the dilute nitride system, which is very attractive for optoelectronics applications, several studies on their electronic and band structure properties were presented.

Symposium Support: AFOSR and AFRL.

\section{State-of-the-Art Nanofabrication Technology Reveals New Paradigms Spanning Research to Manufacturing}

Symposium C, Innovations for Sub100-nm Lithography: Materials and Processes, brought together researchers engaged in developing and advancing state-of-the-art nanofabrication technologies covering a diverse range of lithographic and patterning methods. Since applications include both high-volume manufacturing and specialized sample preparation, many exciting approaches are being developed and explored. Selfassembly concepts were presented, including the use of block copolymers (P.F. Nealey, Univ. of Wisconsin) and "molecular rulers" (P.S. Weiss, PSU), providing ideas for combining "top-down" with "bottom-up" assembly. Current semiconductor fabrication technologies face different and difficult challenges, with materials issues ranging from chemically amplified photoresists (D.L. Goldfarb, IBM; V.M. Prabhu, NIST; C.L. Henderson, Georgia Tech.) to immersion lithography (M. Switkes, MIT Lincoln Lab.) and EUV technology (A. Hassanein, ANL). Nanoimprint lithography shows great promise, particularly for the fabrication of 3D structures (S. Pang, Univ. of Michigan). However, many significant materials science issues remain (C.L. Soles, NIST). Scanning probe and direct-write methods are being developed for specialized applications with advancements including alternative tip materials (H. Zhang, NanoInk) or thermal control (P.E. Sheehan, NRL).

Of additional value was a joint session with Symposium GG, Mesoscale Architectures from Nano-Units: Assembly, Fabrication, and Properties. Perspectives 
on the future of nanofabrication technology for both economically viable manufacturing (semiconductors) and emerging applications were provided. Although nanofabrication in general is a focus within several diverse fields and communities, this session aimed to provide a single forum to summarize and speculate on different approaches. Interesting contrasts in perspective arose, such as those between the semiconductor industry (R.R. Dammel, AZ Electronic Materials), where the focus is on economically viable, large-volume manufacturing methods, and developers of technologies like dip-pen nanolithography (C.A. Mirkin, Northwestern), which aims to provide unique capabilities for the patterning of biological molecules.

Symposium Support: Applied Materials and NIST Office of Microelectronics Programs.

\section{Scaling Issues Drive Materials Research for Nonvolatile Memories (See MRS Proceedings Volume 830)}

Symposium D on Materials and Processes for Nonvolatile Memories brought together scientists working on different memory technologies. A. Fazzio (Intel) extensively reviewed past and expected future trends in flash memory technologies and presented perspectives on new nonvolatile memory architectures beyond 2010. C. Clementi (STMicroelectronics) emphasized flash memory technology development; J. Åckerman (Freescale Semiconductor) reviewed the status of MRAM, focusing on a novel cell and programming method allowing significant improvements; Y. Fujisaki (Hitachi) and J.-P. Han (NIST) reviewed FRAM, with an emphasis on materials and issues to be addressed for nonvolatility; T. Hiramoto (Univ. of Tokyo) and P. Dimitrakis (NCSR, Greece) reviewed the nanoparticle memory field, focusing on integration issues; H. Jeong (Samsung) provided an overview of memory architectures with an assessment of their prospects; and Y. Yang (UCLA) reported on new findings in hybrid organic-inorganic memories.

Many presentations dealt with nanoparticle memory architectures and properties as well as research on understanding and controlling nanoparticle formation during the self-assembly process, which is critical for avoiding statistical distribution fluctuations. New tunneling and control oxides have been introduced in the memory stack of the nanoparticle memory. The FeRAM presentations mainly focused on materials developments and their potential integration with silicon technology, as well as with other inorganic and organic semiconductors. A session was also devot- ed to cross-point memories that are basically of a resistive type and include a sandwich of materials between two conductive electrodes. This architecture, being potentially the most scalable, was the subject of presentations dealing with either new materials, including nanoparticles within a semi-insulating matrix, or with the more elaborate concept of phasechange materials.

Symposium Support: Freescale Semiconductor, Micron Technology Foundation, and Spansion.

\section{LED Progress Marches on, Despite Uncertainty in InN Bandgap (See MRS Proceedings Volume 831)}

The title of Symposium E on GaN, AlN, InN, and Their Alloys reflects the rapid progress made in connecting $\mathrm{GaN}$ at a bandgap of $3.4 \mathrm{eV}$ both to AlN with a bandgap of $6.2 \mathrm{eV}$ and to InN with a bandgap energy around $0.7 \mathrm{eV}$. The fact that a definite value for InN has not yet been agreed upon reflects one of the highlights of this symposium. The previously held value of $1.9 \mathrm{eV}$ has become subject to re-evaluation recently, and the subject has since guaranteed lively scientific discussion at any nitride meeting, including this Fall's MRS Meeting, its long-standing anchor event. Progress in InN has been substantial, and A. Yoshikawa (Chiba Univ.) now reports InN layers grown by MBE that reach a very large thickness of 5-8 $\mu \mathrm{m}$, making them useful for devices. In N-polarity growth, they reach a growth temperature as high as $600^{\circ} \mathrm{C}$.

In the category of GaInN alloys, for example, blue LEDs on 6 in. Si(111) wafers were demonstrated by H.X. Jiang (Kansas State). Jiang's group optimized an AlN nucleation layer to achieve crack-free $\mathrm{GaN}$ layers as thick as $3 \mu \mathrm{m}$. On sapphire-based material, Y. Kawakami (Kyoto Univ.) presented near-field scanning optical microscopy of the light emission in blue GaInN/GaN LED-type quantum wells and identified island-like structures with diameters of 20-70 nm. In time-resolved spectroscopy, he developed a recombination model of localized photocarriers. The role of dislocations and $\mathrm{Mg}$-initiated pyramidal defects on the reliability and performance of laser diodes was thoroughly discussed by S. Tomiya (Sony), and V. Bousquet (Sharp Labs.) reported the improvement of MBE-grown $405 \mathrm{~nm}$ laser diodes with a new low threshold current density of $6.7 \mathrm{kA} / \mathrm{cm}^{2}$ (pulsed) at a threshold voltage of only $8 \mathrm{~V}$.

An area of research receiving a lot of coverage in the Symposium was growth along nonpolar crystal orientations to avoid polarization effects. Here, several groups reported a reduced indium incor- poration efficiency in GaInN layers; for example, O. Brandt (Paul-Drude-Institut) has found significant In surface segregation for the case of $m$-plane growth in MBE. M. Funato (Kyoto Univ.), however, found higher internal quantum efficiency in GaInN/GaN MQWs at $400 \mathrm{~nm}$ over that of polar growth. S. Kamiyama (Meijo Univ.) investigated several nonpolar substrate alternatives: $\mathrm{ZrB}_{2}$, (30̄38) $4 \mathrm{H}-\mathrm{SiC}$, and $r$-face sapphire.

Toward the AlGaN side, the subject of UV light-emitters was dominated by 250-340-nm-wavelength devices. A. Khan (Univ. of South Carolina) found a strong reduction in defect density in $\mathrm{AlGaN}$ by use of a migration-enhanced growth method. In this way, Khan's group achieved output powers of $1.5 \mathrm{~mW}$ at $265 \mathrm{~nm}$, and $3 \mathrm{~mW}$ at $280 \mathrm{~nm}$. M. Crawford (SNL) demonstrated a record toward the shortest-wavelength LED, emitting at $237 \mathrm{~nm}$. Progress in UV-pumped white LEDs was reported by N. Shibata (Toyoda Gosei) using $385 \mathrm{~nm}$ LEDs and multiple phosphors emitting at wavelengths of $450 \mathrm{~nm}, 515 \mathrm{~nm}, 575 \mathrm{~nm}, 560 \mathrm{~nm}$, and $625 \mathrm{~nm}$. In various combinations, Shibata's group achieves high color rendering, a small color shift with drive current, and a large viewing angle.

The subject of spintronics in the nitrides was expertly introduced by T. Dietl (Polish Academy of Sciences) and provided the base for a range of studies on transition-metal-doped GaN.

Research in Group III nitride nanowires and nanowire devices also received a lot of coverage. L.-C. Chen (Natl. Taiwan Univ.) presented the formation and controllability of dislocation-free AlN, GaN, and InN nanowire structures. Among other promising approaches, Y. Li (Harvard) demonstrated radial $n-\mathrm{GaN} /$ $\mathrm{GaInN} / p$-GaN heterostructure nanowires with Mg-doped GaN as the outer shell. This group demonstrated a nanowire blue LED with a peak wavelength of $456 \mathrm{~nm}$.

Symposium Support: Aixtron AG, Cree Inc., Gatan Inc., Lucent Technologies, and SVT Assoc.

\section{Overview Given of the Materials Science and Applications of $\mathrm{Si} / \mathrm{Ge}$ Nanostructures}

(See MRS Proceedings Volume 832)

Symposium F on Group IV Semiconductor Nanostructures reviewed the latest developments in the materials science of $\mathrm{Si}, \mathrm{Ge}$, and SiGe nanostructures. State-ofthe-art techniques to fabricate well-passivated nanocrystals, multilayer Si/Ge quantum wells (E. Fitzgerald, MIT; and groups in the UK and Japan), SiGe nanoclusters (F. Schaffler, Linz; J.M. Baribeau, NRC, Ottawa ; K. Wang, UCLA), and 
single-crystal Si and Ge nanowires (S. Takeda, Osaka) with an aspect ratio (length to diameter) of close to 100 were described. Vertically and laterally ordered $\mathrm{Si}$ and Ge nanostructures were formed using self-organization processes, original growth mechanisms, and new lithographic techniques. A session of the symposium was devoted to complex photonic structures that permitted efficient silicon-based integrated optics to be built (S. Janz, NRC, Ottawa; D.J. Norris, Univ. of Minnesota).

Several theoretical (A. Efros, NRL; A. Willamson, LLNL) and experimental (T. Izumi, Tokai Univ.; C. Reynaud, CEA; A. van Buuren, LLNL) presentations addressed the important physical factors that govern the luminescence properties of Si nanocrystals, such as the presence of shallow impurities and surface defects. It was shown that their light-emitting properties could be enhanced by coupling the excitons with surface plasmons in metal films (J. Biteen, CalTech; A. Polman, FOM

\section{International Materials Research Collaborations Funded by Government}

The key to enabling international materials research collaboration is "mobility," according to a panel on government funding of research. During the International Collaboration Forum held Monday evening, representatives from funding agencies in the United States, Europe, and Mexico described their programs that support these collaborations in both curiosity- and mission-driven projects.

Funding from the U.S. National Research Foundation includes mobility support for students and junior scientists as well as support for collaborative research efforts. Carmen Huber of the Division of Materials Research said that NSF supports curiosity- and/or applications-driven research. NSF has established six International Materials Institutes, including the U.S./Africa Materials Institute located at the Princeton Materials Institute. It is a virtual institution that promotes collaborations between scientists and engineers in the United States and their counterparts in Africa. Wole Soboyejo, who is the project director, described the two focuses of the Institute: (1) advanced materials/small structures and (2) materials for societal development. The Institute also created eight materials education modules.

\section{CONACYT \\ www.conacyt.mx \\ Department of Energy (DOE) \\ www.science.doe.gov/bes/ \\ European Union Research and Development \\ europa.eu.int/comm/research}

Homeland Security Advanced Research Projects Agency (HSARPA)

www.dhs.gov

National Institute of Standards and Technology (NIST) www.nist.gov

National Institutes of Health

Bioengineering Consortium

www.becon.nih.gov

National Institute of Biomedical Imaging and Bioengineering (NIBIB)

www.nibib.nih.gov

National Institute of Dental and Craniofacial Research (NIDCR)

www.nidr.nih.gov

National Science Foundation (NSF)

www.nsf.gov

Office of Naval Research (ONR)

www.onrglobal.navy.mil

U.S./Africa Materials Institute

usami.princeton.edu
Nicolas Newman, advisor for the European Union's International Aspects of Mobility, discussed Europe's progress on supporting researchers' mobility once they arrive in the EU or associated countries. A mobility policy, by way of a "researcher's visa," is being developed in order to resolve current obstacles to traveling between countries. Among the research priorities in Europe are nanotechnology, intelligent materials, and new production processes.

In Mexico, the funding agency CONACYT fosters private R\&D and strategic academic alliances between Mexican and non-Mexican institutions, encourages state-of-the-art research (both basic and applied), and boosts new high-value-added businesses (focusing on technology transfer and technology development). The agency also provides seed funding for collaborative research projects.

Unlike NSF, the U.S. Office of Naval Research supports narrow, integrated, use-inspired materials research focused on Navy and Marine Corps needs. Internationally, said Lawrence T. Kabacoff of the Materials Division, ONR supports U.S. researchers' visits outside the United States and enables nonU.S. technologists contact with their counterparts in the U.S. Department of the Navy.

Nationally, the U.S. Department of Homeland Security also supports materials research and development that is missionoriented, with the main focus on rapid prototyping and commercial adaptation, said Jane A. Alexander, deputy director of the Homeland Security Advanced Research Projects Agency. Robert J. Gottschall, team leader for Materials and Engineering Physics at the U.S. Department of Energy, emphasized that core grants programs in his division are idea-driven. He also introduced the five Nanoscale Science Research Centers that are now in various stages of construction. Likewise, representatives from the National Institute of Standards and Technology discussed their agency's role in the National Nanotechnology Initiative. Clare Allocca and Douglas Smith said that NIST recently assessed the needs and priorities for nanomechanics, for which issues of standardization, calibration, and modeling of nanomechanical experiments came out on top. A roadmap is being drafted for release this year.

Unique research opportunities in nanotechnology are also available in the National Institute of Dental and Craniofacial Research within the National Institutes of Health, said Elena Kousvelari. Furthermore, said Christine Kelley of the National Institute of Biomedical Imaging and Bioengineering, her institute houses research on materials and has a current budget of \$280 million. Kousvelari cautioned that with the completion of the doubling of funding for NIH, the budget is tight and grants are very competitive.

The forum on funding for international collaborations was organized by the Materials Research Society's International Relations Committee. Some of the presentation notes can be accessed from the MRS Web site. More information on the funding agencies can be obtained from their Web sites. 
Inst.). Many presentations dealt with the energy transfer from Si nanocrystals to neighboring light-emitters such as other nanocrystals, oxygen molecules (D. Kovalev, Munich), and erbium impurities (M. Fujii, Kobe; P. Pellegrino, Barcelona; H. Isshiki, Univ. of Electro-Communications; T. Gregorkiewicz, Amsterdam), paving the way to promising optical applications.

Charge transport and the thermoelectric properties (P. Yang, UC-Berkeley) of Si nanowires were discussed, addressing issues concerning the electron coherence and doping efficiency. Charge injection in Si nanocrystals was investigated using scanning probe techniques on single particles (T. Mélin, CNRS) and electrical transport measurements in arrays of nanocrystals and memory devices.

Novel device applications ranging from the first examples of $\mathrm{SiGe} \mathrm{THz}$ lasers (A. Borak, PSI; J. Kolodzey, Univ. of Delaware) and optical gain in Si nanocrystals (L. Pavesi, Univ. Trento, Italy) to prototypes of commercial Si nanocrystal memories (R. Rao, Freescale Semiconductor) were presented.

Symposium Support: Freescale Semiconductor and Hamamatsu.

\section{High-Frequency Devices Pose Special Materials Challenges (See MRS Proceedings Volume 833)}

High-frequency devices pose special challenges in the areas of materials selection, integration, and packaging. The pur- pose of Symposium G was to bring together key workers in the field in order to advance the state of the art in these crucial areas of development. The session began with a discussion of the current science and technology of dielectric films and their integration into high-frequency devices (O. Auciello, ANL). W. Nothwang and colleagues (ARL) discussed barium strontium titanate thin films, with an emphasis on affordable large-area manufacturing that meets industry standards.

In the area of resonators and measurement techniques, P. Muralt (EPFL) discussed the significant challenges faced in the manufacture of BAW resonators based on AlN thin films. The presentation by H. Ohsato (Nagoya Inst. of Tech.) transitioned the symposium to the area of microwave materials. This talk discussed materials with high quality factors and low dielectric constants for microwave devices in wireless communications. The session concluded with J. Dutta (North Carolina Central Univ.) discussing measurements on dielectrics for low-loss windows in the millimeter wave regime.

Talks concerning passive devices ranged from thick-film capacitors (W. Borland, Dupont) to dielectric thin-film components (S. Trolier-McKinstry, PSU). Further talks in this area focused on ferrite thin films, such as those by Y. Takamura (UC-Berkeley) and G. Dionne (MIT Lincoln Lab.). The symposium concluded with a session on MEMS and integration issues and one on ceramics materials and

\section{Research Tools Seminars Introduce Products that Meet Technical Challenges}

As a complement to the 2004 MRS Fall Meeting, the Society offered Research Tools Seminars in its educational program focused on the scientific bases and practical application of commercially available, state-of-the-art tools for materials research. Held in the exhibit hall and free of charge to meeting attendees, these onehour seminars described technical approaches to meeting particular challenges. For example, Thomas F. Kelly, an authority on microstructural characterization from Imago Scientific Instruments Corp., explored 3D atomic-scale compositional imaging using Imago's LEAP technology. He discussed the significance of these developments for a wide range of materials studies, including magnetic storage and semiconductor applications. Other seminars featured tools from Keithley Instruments, Lake Shore Cryotronics, Micro Materials Ltd., and Veeco Instruments.

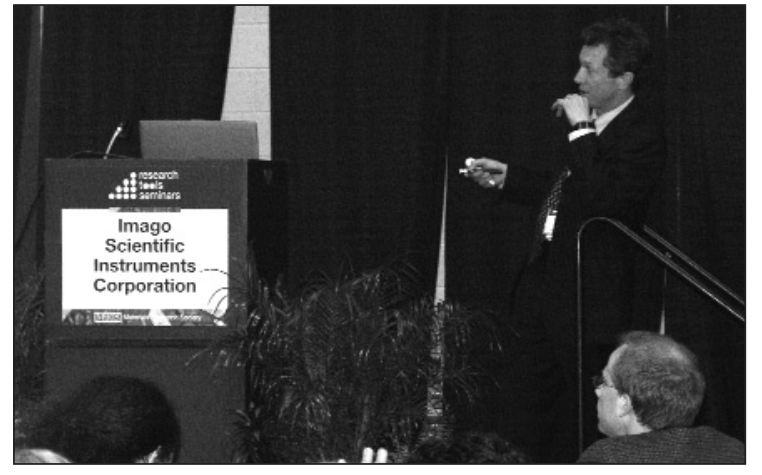

A series of Research Tools Seminars was held in the exhibit hall. packaging. R. Plana (LAAS-CNRS) discussed SiGe technologies for rf and millimeter waves, with J. Tuovinen (Millilab) providing the important links to communications and sensor technology in this frequency regime. T. Vanderash (NIST) concluded with the current needs and opportunities for ceramic materials in wireless communications.

Symposium Support: ARO.

\section{Field of Multifunctional Oxides Blossoms}

"Just five years ago, all of the people working in the field of multiferroics could fit into my office," began N.A. Spaldin (UCSB), the lead-off speaker in Symposium $\mathrm{H}$ on Functional and Multifunctional Oxide Thin Films. But as was evident from the exhilarating results presented in this symposium and the crowded poster sessions, this field addressing materials that are simultaneously magnetic and ferroelectric is exploding. The exceptional properties of functional and multifunctional oxides, combined with the inability of simpler materials to meet the increasing demands of the electronics industry, have motivated tremendous interest and activity in, and emerging technological applications for, these oxides. The symposium showcased exciting results ranging from theory to synthesis, characterization, and applications.

Multiferroics are obscure yet multitalented materials, and Spaldin explained why simultaneously achieving ferroelectricity and ferromagnetism (or ferrimagnetism) is so difficult. She presented firstprinciples results on the multiferroics $\mathrm{BiMnO}_{3}$ and $\mathrm{BiFeO}_{3}$, which were the topic of many experimental presentations that followed, and predicted that ordered superlattices of $\mathrm{BiFeO}_{3} / \mathrm{BiCrO}_{3}$ or $\mathrm{BiMnO}_{3} / \mathrm{BiNiO}_{3}$ would be multiferroic. Several researchers (K.-Y. Yun, Osaka; R.R. Das, Univ. of Wisconsin) reported remanent polarizations, $P_{\mathrm{r}}$, in $\mathrm{BiFeO}_{3}$ in excess of $120 \mu \mathrm{C} / \mathrm{cm}^{2}$, as well as enhanced ferrimagnetism in $\mathrm{BiFeO}_{3}$ thin epitaxial films (R. Ramesh, UC-Berkeley; K.-Y. Yun, Osaka).

Assessing the effect of size and strain on ferroelectricity was a theme in which worldwide progress was reported, including important ramifications for emerging applications. Using photoelectron diffraction, P. Aebi (Neuchatel) saw that $\mathrm{PbTiO}_{3}$ thin films down to $20 \AA$ in thickness lack inversion symmetry, which is a necessary condition for ferroelectricity. S.K. Streiffer (ANL) showed that $\mathrm{PbTiO}_{3}$ films as thin as $12 \AA$ contained ferroelectric stripe domains. Extremely small ferroelectric 
domains were written in ferroelectric films using an AFM approach by P. Paruch (Geneva) and Y. Hiranaga (Tohoku Univ.), achieving storage densities as high as 1.5 Tbit/in. ${ }^{2}$. Biaxial strains of $\sim 1 \%$ in thin commensurate $\mathrm{BaTiO}_{3}$ films grown on $\mathrm{DyScO}_{3}$ substrates were shown by K.J. Choi (Univ. of Wisconsin) to nearly triple $P_{\mathrm{r}}$ and increase $T_{\mathrm{c}}$ by about $500^{\circ} \mathrm{C}$.

Symposium Support: Swiss Natl. Ctr. of Competence in Research and Materials with Novel Electronic Properties-MaNEP.

\section{Patterning of Nanomagnetic Materials Explored}

Symposium I brought together experimentalists working in nanomagnetics. Speakers highlighted both fundamental physical and chemical phenomena related to nanomagnetic fabrication as well as new challenges for nanomagnetic structures, device fabrication, and applications.

A presentation from a Toshiba group showed the possibility of making patterned disk media by using a mask developed from a self-assembled copolymer technique. C. Ross (MIT) patterned small nanorings (100 $\mathrm{nm}$ in diameter) with magnetoresistive measurement. New results on the fabrication of a magnetic nanotube with a diameter of $\sim 100 \mathrm{~nm}$ was presented by K. Nielsch (MPI) and D. Sellmyer (Univ. of Nebraska). G. Hadjipanayas (Univ. of Delaware) and D. Sellmyer (Univ. of Nebraska) fabricated nanomagnetic particles, with uniform sizes ranging from $3 \mathrm{~nm}$ to $10 \mathrm{~nm}$, embedded in a nonmagnetic matrix by multilayer sputtering for magnetic media application. J.-P. Wang (Univ. of Minnesota) demonstrated a fullfunction programmable magnetic logic device using a single MTJ cell. The latest progress in nanobiomagnetic detection was given by G. Reiss (Univ. Bielefeld) and S. Wang (Stanford). Detection of several tens of magnetic oxide nanoparticles with sub-20-nm diameters by GMR and MTJ sensors was demonstrated. Use of a new spintronics switching mechanism (spin transfer) for MRAM applications was demonstrated by R. Buhrman (Cornell) and Y. Huai (Grandis).

Symposium Support: Heraeus.

\section{Magnetic Photonic Crystals Highlighted (See MRS Proceedings Volume 834)}

Symposium J, Magneto-Optical Materials for Photonics and Recording, highlighted recent developments in both materials research and device fabrication for a wide variety of applications. One subject receiving a lot of attention was that of magnetic photonic crystals. M. Inoue (Toyohashi Univ. of Tech.) presented results on the fabrication and testing of
$\mathrm{SiO}_{2} / \mathrm{Bi}$ YIG (yttrium iron garnet) magnetophotonic crystals. By applying a magnetic bias field along the axis of optical propagation, Inoue demonstrated simultaneous high optical transmission and large Faraday rotation in a thin-film, 1D magnetophotonic crystal. His system includes an extra-long central Bi:YIG layer, which forms an effective Fabry-Perot cavity, leading to concentration of optical intensity in this layer and thus enhanced magnetooptical effects. Inoue is also investigating 2D magnetophotonic crystals based on $\mathrm{Al}_{2} \mathrm{O}_{3}$ templates and Bi:YIG rods and 3D magnetophotonic crystals using a selforganizing technique. This technology is likely to have important applications for magneto-optical devices, including optical isolators and spatial light modulators for holographic memories.

In the next talk, A. Figotin (UC-Irvine) discussed the use of magnetic photonic crystals as artificial magnetoelectric media. Unlike their dielectric counterparts, magnetophotonic crystals (MPCs) can display non-reciprocal spectral properties, including asymmetric dispersion relations and unidirectional propagation of light. Figotin said that both time-reversal and space-inversion symmetry must be lacking in a magnetophotonic crystal for it to display non-reciprocal properties. He predicted the existence of a "frozen mode," in which the group velocity of an optical pulse in a 1D magnetophotonic crystal with three-layer unit cells is large in one direction and nearly zero in the opposite direction. The frozen mode regime can display very little pulse reshaping and could be useful for optical isolation or optical pulse storage.

Other topics of current interest include techniques for creating photonic bandgaps, as well as resonant transmission and an enhanced Faraday response in magnetooptic crystals (A. Grishin and S. Kahl, (Royal Institute of Technology, Sweden; M. Levy, Michigan Tech. Univ.; B. Stadler, Univ. of Minnesota). C. Flytzanis (Ecole Normale Supérieure, Paris) discussed the photoinduced gyrotropy in semimagnetic semiconductor magnetophotonic microcavities, and S. Nikitov (Russian Acad. Sciences, Moscow) in collaboration with C. Tsai (UC-Irvine) presented the fabrication and testing of 2D magnonic crystals.

Several advances in technologies required for practical device applications were also reported. N. Imaizumi (Namiki Precision Jewel Co., Tokyo) discussed new garnet thick films with a very low temperature dependence of the Faraday effect. Methods to integrate magneto-optical devices with semiconductor devices were demonstrated, including a direct bonding technique described by T. Mizumoto (Tokyo Inst. Tech.) to make waveguide isolators. V. Zayets (AIST, Japan) used the diluted magnetic semiconductor (Cd,Mn)Te as a magneto-optical waveguide grown on a GaAs substrate to obtain a complete transverse electric to transverse magnetic waveguide mode conversion. H. Shimizu (Univ. of Tokyo) used a hybrid structure of a ferromagnetic metal and semiconductor optical amplifier to obtain $93 \mathrm{~dB} / \mathrm{cm}$ isolation. This hybrid structure can also work as an optical transistor and high-speed optical memory, as shown theoretically by Zayets.

Magneto-optic materials for recording applications were another highlight of the symposium. By fabricating multilayer thin-film stacks of magnetic materials, coupled either by means of their magnetostatic or exchange interactions, it was shown that storage densities could be substantially increased. A. Itoh (Nihon Univ.) discussed a means of doing this by using multiple storage layers, while both T. Miyakoshi (Canon) and H. Awano (Hitachi Maxell) discussed domain-wall displacement detection and zero-field magnetic amplifying magneto-optic system methods, respectively, that enable read-back of magnetic domains that are much smaller than the diffraction limit.

Heat-assisted magnetic recording (HAMR) is a topic that is new to this symposium. A tutorial on this subject was presented by T.E. Schlesinger (CMU). Although one might suppose that switching times in ferromagnetic HAMR media might be limited by the ferromagnetic resonance frequency, experimental results presented by A. Tsukamoto (Univ. Nijmegen, Netherlands) and R. Van de Veerdonk (Seagate Technology) clearly demonstrated a much faster switching rate at elevated temperatures that should easily support GHz data rates. The mechanism for this fast response time, however, is unclear. It was also shown by J. Thiele (Hitachi Global Storage Technologies) that the novel magnetic characteristics of exchange-coupled thin films of the ferromagnet FePt and ferromagnet/antiferromagnet FeRh are precisely what is needed for stable HAMR media.

Symposium Support: Michigan Technological Univ. and Seagate Technology.

\section{Advances Made in Solid Ion Conductors and Materials for Energy Technology and the Environment} (See MRS Proceedings Volume 835)

Symposium K on Solid-State Ionics reported advances in energy storage and conversion technologies, especially lithium-ion batteries, polymer electrolytes (PEMFCs), and SOFCs. Particular high- 
lights of the symposium were joint sessions on solid-state ionics-based chemical sensors (with Symposium A) and solidstate chemistry of ionic conductors (with Symposium FF). Chemical sensors are much needed to improve the efficiency and environmental compatibility of automotive transport; talks from academia and industry showed recent advances in this domain. Another remarkable trend was the development of in situ techniques, including solid-state NMR and the use of synchrotron radiation, to obtain a detailed knowledge of mechanisms.

The sessions on solid ionic conductors and theory showed recent advances in modeling and simulation, especially using $a b$ initio methods, and how the improved understanding can enable the design of better ionic conductor devices. Special interest was devoted to solid proton conductors and oxides for oxygen separation membranes.

In the fuel cell session, a central topic was the improvement of solid ion conductor separation membranes. Several new or improved solid electrolytes were presented. Furthermore, the cathode side of the cell, where oxygen reduction takes place, was heavily investigated.

The largest cluster of the symposium was devoted to lithium batteries. A large variety of contributions was presented, ranging from improved electrode materials and electrolytes to studies of fundamental battery mechanisms. The impressive advances obtained recently by use of nanostructured or nanocomposite electrodes and electrolytes give a major drive to this field.

Symposium Support: UMICORE Research.

\section{Maturing Solar Cell Research \\ Branches out into Organics}

(See MRS Proceedings Volume 836)

Symposium L, Materials for Photovoltaics, brought together discussions of the materials properties and conse-

\section{Scientists and Teachers Forge Partnerships to Advance Materials Education}

Close to one hundred high school science and math teachers selected from across the United States joined materials researchers in Symposium PP, Communicating Materials Science-Secondary Education for the 21st Century, at the 2004 MRS Fall Meeting in Boston. The program was designed to bring together university faculty, researchers, education program administrators, and high school teachers to explore the best collaborative practices for integrating materials science into k-12 classroom teaching. The symposium was preceded by a special program at the Boston Museum of Science, where the MRS Strange Matter exhibition was on display. At the museum, the teachers attended lectures highlighting current materials research and participated in hands-on workshops that provided them with activities for classroom use.

At the opening session of the two-day symposium focusing on materials science literacy, Dennis Bartels, president of TERC in Cambridge, pronounced the need for scientists to serve as "advocate educators." Bartels joined TERC after serving as director of the Center for Teaching and Learning at the Exploratorium in San Francisco. He said that science education must provide both a universal science literacy and a career path. Bartels said that for the past hundred years, the United States developed a policy valuing science literacy for everyone, drawing minorities into the fold. Since 9/11, policymakers have become more interested in creating a channel for science careers, in response to the decrease of science students in U.S. universities from outside the country. Bartels quoted Ann Druyan, who said that the United States needs science literacy in order to preserve democracy. Such literacy, for example, serves as a "science baloney detector." He said that materials researchers can serve as advocate educators by getting curious about how people learn, helping others who are good at translating science (e.g., museums, media, and the Web), and supporting funding for $\mathrm{k}-12$ science education.

Advocacy at the policy level works, as seen when Andrea Harmer announced a commitment made by Governor Ed Rendell of Pennsylvania to make broadband available in all Pennsylvania schools (k-12), which will enhance Web-based education. Harmer, director of Web-Based Education in the Center for Advanced Materials and Nanotechnology at Lehigh University, described a hands-on problem-solving course taught to middle school students, in which they have remote use of Lehigh's electron microscope.

A workshop was held twice during the course of the Meeting, at which Anissa Ramirez (Yale) and Amy Moll (Boise
State Univ.) led hands-on demonstrations in teaching materials science concepts. Six tables were set up, each containing 2-3 activities, including demonstrations on diffraction, ferrofluids, and amorphous metals. At one table, for example, participants created electromagnets by connecting a nail to a $\mathrm{C}$ battery with tiny loops of wire around the nail, which magnetized the paramagnet. After playing with the "toys," the participants regrouped and shared other activities they have done in their classrooms.

Educators who had participated in NSF Research Experience for Teacher programs at universities reported on the integration of their experiences into their curricula. Participants learned about on-line resources, creative classroom demonstrations, the use of remote access to state-of-theart research equipment, and other examples of successful partnerships between teachers and practicing materials scientists.

Separate from the education symposium, a group of students from the Garcia Research Scholar Program for High School Students, located at the SUNY-Stony Brook MRSEC, gave talks and poster presentations based on their research in polymers.

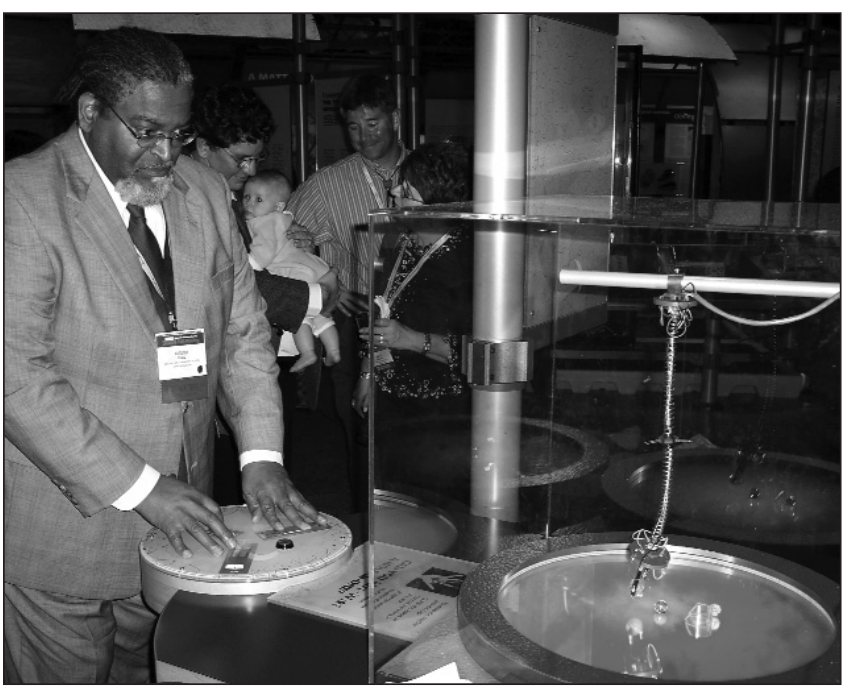

During a visit to the MRS Strange Matter exhibition at the Boston Museum of Science, high school teachers and materials researchers interacted with the displays. 
quences for device applications of a broad range of semiconductor materials. The first half of the symposium was concerned with the emerging fields of organic, polymer, and dye PV cells. S. Forrest (Princeton) described problems and solutions for organic semiconductors in photovoltaics. Short diffusion lengths can be addressed with light-trapping schemes, while high series resistance is reduced by going to OVPD growth.

The second half of the symposium covered new developments and insights in the more traditional semiconductor PV materials. W. Walukiewicz (UC-Berkeley) discussed the possibility of using the InGaN system for photovoltaics, focusing on band structure, doping, and radiationhardness properties of the material. Using photoluminescence intensity as the metric, Walukiewicz reported that $\mathrm{InN}$ is more radiation-hard than $\mathrm{GaN}$, because for $\mathrm{InN}$ the Fermi stabilization energy is outside the bandgap. He also reported p-type doping of InN:Mg, although there is still an $n$-type accumulation layer at the surface. A. Bett (Fraunhofer ISE) discussed lattice-mismatched GaInP and InGaAs for multijunction solar cells. He found GaInP not to be suitable as a graded layer, due to threading dislocations. In contrast, GaInAs serves well as a graded layer, with no difference being observed between stepped and linear grading schemes.

Symposium Support: AFRL and NREL.

\section{Direct-Methanol Fuel Cell Gains Momentum}

In Symposium $\mathrm{M}$ on Materials Aspects of Fuel Cells, S.M. Haile (CalTech) gave an impressive talk on intermediatetemperature electrolytes based on Cs hydrogen sulfates. These fuel cells operate at temperatures where methanol reforming is facile. The prospects of a high-performance direct-methanol fuel cell that beats the power density of hydrogen-air fuel cells is exciting. Haile's fuel cell operates at a temperature where $\mathrm{CO}$ is not toxic, obviating the need for the bulky water-gas-shift reactor and the preferential oxidation units that PEM fuel cells require for the removal of $\mathrm{CO}$. S. Mukerjee (Northeastern) took synchrotron studies of fuel cell catalysis to a new height with a presentation on in situ XAS studies of oxygen reduction, $\mathrm{CO}$, and methanol oxidation. A very sobering talk was given by S. Kocha (GM) in which it appears that the carbon support at the cathode side is vaporizing to $\mathrm{CO}_{2}$ over time, resulting in the ripening of the cathode $\mathrm{Pt}$ catalysts. Although carbon gasdiffusion layers may be usable, Kocha's findings could mean the end of carbon particle supports for the cathode catalysts.

\section{Role of Titanium Catalyst in Hydrogen Storage Debated (See MRS Proceedings Volume 837)}

In Symposium $\mathrm{N}$ on Materials for $\mathrm{Hy}-$ drogen Storage, one session focused on metal hydrides, beginning with an overview by H. Birnbaum (UIUC) on important basic issues of hydrogen storage in metals. This was followed by shorter talks focused on particular alloy systems. J. Reilly (BNL) outlined hydrogen-driven metallurgical reactions and their use to make hydrogen storage materials as well as high-surface-area electrodes for batteries. He also drew attention to the often overlooked fact that DOE's goal to refuel a car within 3-5 min is a formidable challenge due to the heat generated.

Another session focused on the fastmoving area of complex metal hydrides. K. Gross (SNL) gave an overview of the paradigmatic system, Ti-doped $\mathrm{NaAlH}_{4}$. This system, which up to now has been the only reversible complex metal hydride system at moderate conditions, was the subject of further shorter presentations by E. Majzoub (SNL) on its vibrational properties and by J. Graetz (BNL) and Y. Gao (GE) on the nature of the Ti catalyst. A theoretical perspective on this system was given by P. Jena (Virginia Commonwealth), and a lively discussion broke out before and after the talk of $\mathrm{C}$. Jensen (Hawaii Univ.), who presented a tour de force presentation on interdisciplinary collaborations using a plethora of different techniques to pinpoint the role of titanium, which makes this system reversible.

Carbon-based systems and their potential for hydrogen storage were addressed. The exploratory nature of this new and exciting area was well introduced by H. Cheng (Air Products and Chemicals), who presented molecular dynamics simulations on the adsorption and storage of hydrogen in single-walled carbon nanotubes. A. Dillon (NREL) outlined a promising new route to achieving reversible hydrogen storage approaching $9 \mathrm{wt} \%$ by forming complexes between transition metals and aromatic carbon nanostructures. R. Yang (Univ. of Michigan) focused on graphite nanofibers and their potential for hydrogen storage by spillover mechanisms. Other new materials for hydrogen storage, in particular, metalorganic frameworks (MOFs), were discussed by O. Yaghi (Univ. of Michigan), who pioneered the synthesis of these materials.

In the area of chemical hydrides, M.-Y. Chou (Georgia Tech) presented first-principles theory of $\mathrm{Ti}$ substitution in bulk alanate storage materials. A number of research groups from the General Motors Research and Development
Center and HRL Laboratories presented results on newly synthesized hydrides containing primarily $\mathrm{Li}, \mathrm{B}$, and $\mathrm{N}$ with a hydrogen weight of between $6 \%$ and $11 \%$ and demonstrating, in some cases, reversible hydrogenation properties. A theme in these materials seems to be to pick the thermodynamically accessible stages of a multistep decomposition and to have materials with a complex phase mixture. Again, Ti has been used to enhance the kinetics with limited knowledge on how it works.

Symposium Support: NREL and UIUC.

\section{Scanning Probe Microscopy Advances Study of Electronic, Mechanical, and Optical Properties \\ (See MRS Proceedings Volume 838)}

Symposium $\mathrm{O}$ served as a focal point for developers and advanced users of SPM and related techniques. Investigations and tools to deduce electronic, mechanical, and optical properties were described from atomic to micrometer length scales. On Thursday afternoon, E.W. Plummer (ORNL) led a panel discussion among representatives from academia, national laboratories, and funding agencies. The panel addressed visions of and mechanisms for future SPM advancement. While perspectives varied between disciplines, there was general agreement on three key points: (1) continued development in SPM instrumentation and theory is important both to improve present capabilities and to drive future discovery; (2) the cost and complexity of building and sustaining new SPM technologies are a challenge for academia and industry, based on present funding opportunities; and yet, (3) the SPM community has been remarkably successful in distributing basic equipment and knowledge such that SPM is now a nearly ubiquitous tool.

Symposium Support: Asylum Research, Molecular Imaging, Nanotech-America, ORNL, Omicron NanoTechnology USA, PSIA, RHK Technology, and Veeco Instr.

\section{Electron Microscopy Probes Deep Sub-Angstrom Regime (See MRS Proceedings Volume 839)}

The field of electron microscopy has undergone a revolution in recent years, with dramatic improvements in electron optics, image reconstruction algorithms, and novel in situ methods. Symposium P on Electron Microscopy of Molecular and Atom-Scale Mechanical Behavior, Chemistry, and Structure focused on the ways that these advances are having an impact on an understanding of materials and biological systems at the nanometer to sub-angstrom scale. The advent of aber- 
ration correction has yielded image resolutions in the deep sub-angstrom regime. O. Krivanek (Nion, Kirkland, Wash.) reviewed the pathway from the earliest aberration correctors built on a shoestring budget to the present sophisticated C3/C5 correctors that reveal image transfer to below $0.78 \AA$. M. Chisholm (ORNL) showed that the probe corrector enhanced both amplitude and phase contrast images, which could be recorded simultaneously in the STEM geometry, combining the benefits of both methods to reveal the normally complementary heavy and light atom locations. These technological advances in electron optics have moved sufficiently far into the laboratory that they are yielding important results in many fields of materials science, from grain-boundary structure in ceramics (A. Ziegler, LLNL) and complex oxides (P. Prabhumirashi, ORNL) to detailed knowledge of depth-resolved dopant distributions in gate oxides (K. van Benthem, ORNL) and dislocations (N. Browning, LBNL).

Additionally, with the advent of electron tomography, electron microscopy is no longer limited to two dimensions. This symposium brought together experts from biological cryoelectron tomography and condensed-matter STEM to provide an overview of the ways in which 3D electron tomograms are created and used to understand such diverse processes as ribosome function (J. Frank, Wadsworth Center), catalytic activity (M. Weyland, Cornell), and nanoparticle distributions and structures (I. Arslan, Cambridge) and how they can be exploited in semiconductor failure analysis (C. Kuebel, FEI).

Finally, novel in situ studies exploring the interrelationship between structure and properties were presented. R. DuninBorkowski (Cambridge) showed images of internal dopant fields of semiconductor devices during operation using electron holography, J.P. Cumings (Stanford) reviewed how scanning probes interfaced to the TEM can be used to measure the electronic and mechanical properties of nanotubes. F.M. Ross (IBM Watson) reviewed her recent work on understanding electrodeposition through exploitation of liquid cell experimentationimaging nanoscale nucleation from a liquid in the electron microscope. It was a stimulating session, in keeping with the exciting times in this field.

Symposium Support: Delong Instr. USA, E.A. Fischione Instr., FEI, and JEOL USA.

\section{Scattering Techniques Facilitate Characterization of Hard and Soft Matter}

\section{(See MRS Proceedings Volume 840)}

The overall aim of Symposium Q on Neutron and X-Ray Scattering as Probes of Multiscale Phenomena was to bring

\section{Scientists Discuss Workforce Issues Facing Women in Academia}

Early Wednesday morning, materials scientists gathered for a panel discussion on Workforce Issues Facing Women in Academia: Developing a Career and a Lifestyle. The panel was featured at the Women in Materials Science and Engineering Breakfast sponsored by the MRS Public Outreach Committee, Aldrich Chemical Co., and Veeco Instruments. In a question-and-answer format, the panelists, Gillian Bond (New Mexico Tech), Lorna Gibson (MIT), Ingrid St. Omer (Univ. of Kentucky), and Linda Vanasupa (CalPoly) described their experiences handling career issues with the personal backdrop of dual-career households, households with children, and as minorities in a department or research program. Panelist Larry Pope (SNL) provided some insight from a hiring point of view, and the moderator, Sandy Yulke (Yale), offered career options in academia other than teaching and research. Through the various experiences described by the panelists and attendees, the main criteria for success seem to be mutual support for everyone's career within the family, a geographical location in which the region offers many resources for career advancement, and flexibility in career decisions so as to match career needs with the right "boss." Above all, when individuals perform well, they will find institutions that want them.

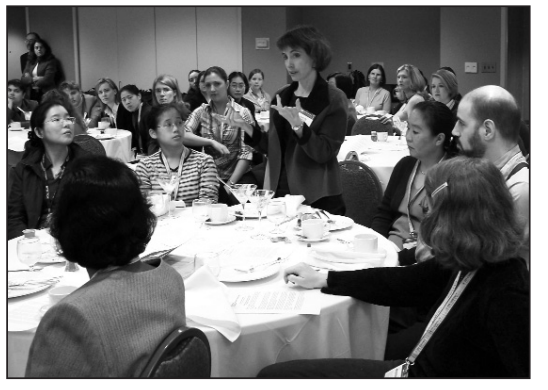

During a breakfast program, attendees engaged in a discussion with panelists on workforce issues facing women in academia. together technical experts and users of recent advances in instrumentation (e.g., ultrasmall-angle neutron and x-ray techniques, inelastic neutron scattering) in order to discuss cutting-edge scattering techniques for the characterization of hard and soft materials. These new techniques, coupled with worldwide investment in powerful radiation and particle sources (e.g., intense synchrotron sources and spallation neutron sources), have greatly expanded the capabilities and user base of scattering techniques, with a profound impact on the materials community. The research discussions introduced a broader materials community to the utility of $x$-rays and neutrons in the characterization of materials.

A good example of the use of new scattering techniques in the characterization of soft materials was the discussion of inelastic neutron scattering by A. Sokolov (Univ. of Akron) to characterize dynamics in biopolymer solutions. His research has found that stability in frozen proteins is heavily dependent on the dynamics of encapsulating water around the protein, dynamics not accessible prior to the development of neutron backscattering spectroscopy.

Cutting-edge $\mathrm{x}$-ray techniques are also being used to examine strain-related phenomena. J. Budai (ORNL) described the development of a novel microdiffraction $x$-ray technique to examine $2 \mathrm{D}$ and $3 \mathrm{D}$ structural studies of materials on mesoscopic length scales of tenths to hundreds of microns. For example, this technique can be used to obtain a map of grain orientations and grain-boundary motion in polycrystalline aluminum during 3D thermal grain growth. Related microdiffraction techniques can be used to probe structure, plasticity, and fracture in thin metal films, as described by R. Spolenak (MPI, Stuttgart). These types of measurements can be combined with anomalous diffraction and coherent diffraction to characterize displacement fields in thin films and multilayers.

\section{Characterization of Dynamic Mechanical Properties Emphasized in Fundamentals of Nanoindentation and Nanotribology}

(See MRS Proceedings Volume 841)

Symposium R, Fundamentals of Nanoindentation and Nanotribology, brought together a wide range of researchers focused on everything from molecular dynamics simulations of nanoasperity contacts to nanomechanical characterization of hard tissues. The diverse range was highlighted by two joint sessions on Nanomechanics and Tribology of Bio- 
logical Materials (with Symposium Y) and Indentation and Phase Transformations (with Symposium T).

Nanoindentation has matured into the standard method for characterizing mechanical behavior in small volumes. However, there is now substantial interest in extending the range of materials to which it can be applied. In particular, polymeric materials and biological systems have become of enormous interest. These materials often exhibit time-dependent properties, and consequently, the standard methods for analysis are not always appropriate. This was highlighted during two sessions dedicated to advances in instrumentation and the examination of timedependent properties. These featured talks on new experimental methods by W. Oliver (MTS Corp.), O. Warren (Hysitron), and A. Fischer-Cripps (CSIRO, Australia). In addition, the potential of nanoindentation and its current limitations in the characterization of viscoelastic materials were highlighted by $\mathrm{H}$. Lu (Oklahoma State), C. White (NIST), J. Houston (SNL), and M. Van Landingham (ARL). An informal evening panel discussion on time-dependent behavior was chaired by T. Page (Newcastle Univ.). This demonstrated the vibrancy of research in the area and highlighted the potential for new breakthroughs in time-dependent nanomechanical testing.

In the area of nanotribology, the symposium included one of the Fall Meeting's highlights, a presentation by Jacob Israelachvili, recipient of an MRS Medal. Israelachvili presented some of his key findings from more than 30 years of research into surface and intermolecular forces. This was part of an exciting morning and afternoon session dedicated to studies of frictional forces and wear.

Symposium Support: GM RED Ctr. and Hysitron.

\section{Underlying Mechanisms in Intermetallic Compounds for Structural and Functional Applications Revealed (See MRS Proceedings Volume 842)}

The overarching theme of Symposium S was the critical importance of microstructure and defects on the physical and mechanical properties of ordered intermetallics. These compounds have great promise for a variety of diverse and important applications such as high-temperature structural materials, "smart" shape-memory alloys, hydrogen storage media, thermoelectric power sources, and magnetic applications. The integrative aspects of this topic were highlighted to encourage a common platform for the presentation of intermetallics

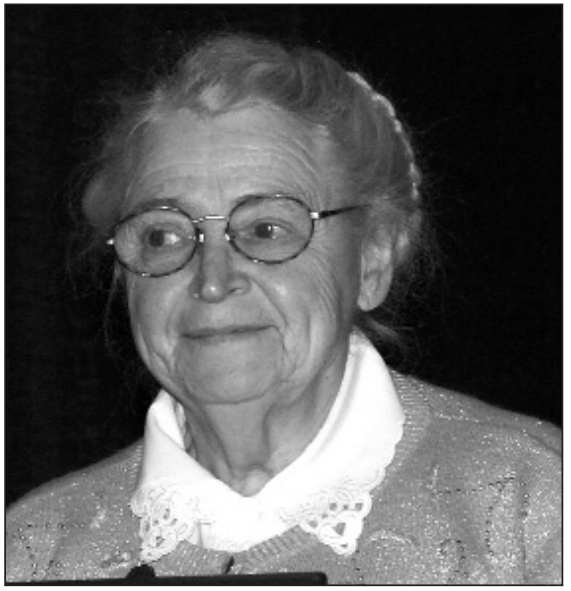

Mildred S. Dresselhaus (MIT) delivers the plenary address on advanced materials for energy.

research that may have different technological backgrounds and objectives, yet in all cases require a sound understanding of the elementary processes that govern structural and functional propertiesfrom the atomistic through microstructural scales. The symposium highlighted the tremendous progress in the field with respect to processing and properties, as well as improved fundamental insights through novel experimentation and modeling activities.

The latest research involving the development of alloy systems with improved high-temperature strength was one of the focal areas of the symposium. For example, alloy systems with microstructures containing large volume fractions of phases such as titanium aluminides, molybdenum aluminides, and niobium aluminides show great promise for extending design windows for aeroengine applications beyond those currently possible with conventional metallic alloys. The iron aluminides exhibit remarkable pseudo-elastic response, with a fully reversible elastic response at room temperature that is much larger than for other metals. The physical mechanism for this effect is being uncovered using novel in situ neutron diffraction studies. It is known that the shape-memory effect in the important nickel titanium system is caused by a series of thermomechanically driven phase transformations. HRTEM has been used as a powerful probe to quantitatively characterize the local internal strains that drive these transformations down to the atomic scale. For hydrogen-storage intermetallics, such as lanthanum nickel compounds, numerous defects including dislocations and vacancies are introduced during hydrogenation. Ways to improve hydrogen storage characteristics by controlling the defect population and arrangement were extensively discussed.

Guiding principles toward improved thermoelectric compounds were also presented. A number of potential materials systems were discussed, and new processing approaches were highlighted, such as nano-superlattice and nanodot formation for which the importance of dimension reduction for phonon and electron scattering control is providing new opportunities.

Symposium Support: Boehler Schmiedetechnik GmbH; JEOL, Japan; Kyoto Univ.; ORNL; and Shimazdu.

\section{Surface Mechanics Shaped by Surface Modification \\ (See MRS Proceedings Volume 843)}

Symposium T covered a broad range of topics related to surface engineering, surface modification, and surface mechanics. The symposium began with an invited talk by C. Mitterer (Univ. Leoben), who reviewed a number of important topics in the field of plasma-assisted vapor-deposited hard coatings. The thermal stability of TiN was shown to be improved by alloying with $\mathrm{Al}$, and $\mathrm{Cl}$-alloyed $\mathrm{TiN}$ was shown to reduce friction coefficients. Also discussed was the potential of the Magneli phases as solid lubricant materials. Additional presentations were made on the use of nitride coatings for cutting tool applications, particularly $\mathrm{AlCrN}$. The state of the art in thermal barrier coatings (TBCs) was discussed in a presentation by D. Zhu (NASA), highlighting the development of a new generation of TBC systems on silicon-based ceramic substrates that will enable long-term improvement in TBC performance and lifetime.

Further research on carbon-based coatings was presented, and it was shown how disordered carbon films with high hydrogen contents can provide nearly frictionless coatings. J.M. Martin (Ecole Central de Lyon) discussed mechanisms of superlow friction in molybdenum disulphide and diamond-like carbon coatings. The symposium then continued with a session on the mechanical properties of surfaces and interfaces, and topics ranged from nanocomposite coatings to solder joints in electronic packages to the tribological applications of shape-memory alloys. Research work on ion-beam modification of surfaces and thin films was discussed by J.M.E. Harper (Univ. of New Hampshire), who reviewed the effects of ion-beam bombardment on the composition, texture, and topology of thin films. The importance and origin of intrinsic stresses in 
thin films was outlined by M. Nastasi (LANL). A joint session was held with Symposium $\mathrm{R}$ that covered indentationinduced phase transformation in Si and Ge. Results of cross-sectional TEM studies highlighted the effects of indenter angle on these transformations, and it was noted that these transformations may facilitate ductile machining of semiconductor materials. An overview of high-pressure surface science and engineering was presented by V. Domnich (Drexel).

The symposium concluded with a session on the synthesis and characterization of surfaces and coatings, including discussions on the synthesis of boron nitride coatings on $\mathrm{SiC}$, and an electron microscopy analysis of white iron surfaces after dry sliding contact, which outlined the microstructural changes induced by dry sliding.

The final talk was on surface modification of polymers by the use of supercritical fluids. This talk was an interesting departure from the usual meeting presentation, in that it was presented by a high school student, Chelsea Gordon, who had worked as a summer intern under the direction of M. Rafailovich (SUNY-Stony Brook). This well-presented talk demonstrated the significant potential and talent that future generations of materials researchers hold.

Symposium Support: Balzers, NSF, and Renishaw.

\section{Morphology and Stability Examined across a Broad Swath of Micro- and Nanostructures}

Symposium U, Stability of Thin Films and Nanostructures, was highly diverse in nature and the first to address related aspects of this broad topic in a single symposium. The overall goal was to bring together scientists working on different materials systems to elucidate phenomena related to morphological and mechanical stability of small-scale structures. The first day of the symposium was devoted to stability of self-assembled surface nanostructures. This session featured six invited speakers who discussed a variety of ways-some demonstrated experimentally, some still theoretical-in which surface structures may be controlled. From surface self-assembly, the symposium moved on to discussions pertaining to the stability of nanoparticles and nanowires. Highlights included discussion of recent discoveries related to sintering of nanoparticles to form metal wires. Stability of microscale films and structures was the next topic. Much of the emphasis on time-dependent mechanical behavior and failure included novel testing methods wherein cyclic or time-dependent loading procedures were used to extract fundamental properties on the nanoscale that are related to creep, viscoelastic behavior, fracture, or fatigue. Exciting recent developments presented in this session included an emerging picture of stress-driven grain growth in thin metal films, the continued advance of reactive bonding toward a feasible joining technology, in situ TEM observations of microtensile testing, and demonstrations of significant viscoelastic behavior in thin metal films. The final session addressed the stability of mostly soft polymeric materials systems. The session focused on the phenomena of wrinkling in bilayer systems of mismatched moduli. Key topics of interest included deformation of nanostructures and discussion of the wetting, stability, and thermodynamics of polymer thin films, explained with combined experimental data and theory for nonequilibrium dynamics.

Symposium Support: ARO.

\section{Plasticity Behavior Depends on Length Scales}

Symposium V, Size Effects in Plasticity, examined the mechanical response of solids under deformation at length scales from the macro- to the nanoscale. Both experimental and theoretical/computation studies focused on identifying processes that govern plastic response at various length scales in different materials as a function of composition, microstructure, deformation geometry, temperature, and strain rate.

Several researchers (J. Greer and W. Nix, Stanford; D. Dimiduk, AFRL; D. Norfleet, OSU) described experiments on uniaxial compression of metal pillars with diameters ranging down to $0.5 \mu \mathrm{m}$, fabricated using a focused ion beam. Nix theorized that observed size effects are due to "dislocation starvation" arising when the sample size falls below the dislocation "breeding distance." This mechanism differs from that proposed to explain size effects in strain-gradient geometries. Size effects were also described in deformation without strain gradients in nanobelts and nanowires (K. Gall, Univ. of Colorado). Meanwhile, K. Van Vliet (MIT) described nanoindentation studies in thin $\mathrm{Cu}$ lines; the increased compliance in the thinner line was attributed to a dislocation mechanism.

Many researchers discussed experimental artifacts that can either mask a small size effect (e.g., surface strains from processing) or give an erroneous size effect (e.g., sample porosity, strain rate effects, or texture evolution). L. Lu and M. Dao, both of MIT, presented experimental and simulation studies of rate effects in nanoindentation of pure $\mathrm{Cu}$ thin films with a high density of twins. P. Anderson (OSU) presented a simulation study of yield and hardening in multilayer thin films; N.S. Weingarten (Catholic Univ.) used atomistic simulation to explore the relationship between size effects and rate effects, showing how geometrically necessary dislocations coalesce to form grain boundaries.

Studies of deformation mechanisms in nanocrystalline materials were presentedand hotly debated - by many researchers. V. Yamakov (Natl. Inst. of Aerospace), $H$. van Swygenhoven (Paul Scherrer Inst., Switzerland), F. Spaepen (Harvard), S. Mao (Univ. of Pittsburgh), D. Farkas (Virginia Tech), Y. Qi (GM), M. Demkowicz (MIT), E. Marquis (SNL), and others presented viewpoints on the mechanisms that control mechanical response and what type of cross-over occurs when grain size drops into the nanometer range. Despite considerable progress in both experiment and simulation, the diversity of opinion remains wide; one presenter compared the situation to the story of the blind men and the elephant. Shock propagation in nanocrystalline metals was discussed by Y. Wang (LLNL) and M. Baskes (LANL).

Several multiscale modeling efforts to connect the atomistic to continuum behavior were aiming at 2D and 3D simulations of real-time behavior at small length scales. W. Curtin (Brown) presented a coupled atomistic-discretized dislocation model which shows $R$-curve (increasing crack resistance) behavior with crack growth. L. Levine (NIST) described a technique for combining first principles with atomistic simulation of nanoindentation. M. Ortiz (CalTech) presented a 3D multiphase field approach and described an energy representation of how a material responds to shearing depending on its controlling length scale and temperature.

Symposium Support: AFOSR and ARO.

\section{Going beyond Actuation with Mechanically Active Materials}

The overall aim of Symposium W, Mechanically Active Materials, was to bring together materials scientists and engineers who focus on a range of distinct materials systems ranging from shapememory polymers to ferroelectric ceramics, in order to identify commonalities in mechanisms and modeling of mechanically coupled (or active) materials. Thermoand electromechanically coupled alloys and composites were demonstrated as viable materials for applications beyond actuation (K. Bhattacharya, CalTech), including Si-based microelectronics 
(H. Atwater, CalTech) and data storage (W. Crone, Univ. of Wisconsin). New approaches to combinatorial synthesis (J. Cui, Univ. of Maryland) and processing (K. Gall et al., Univ. of Colorado) were presented as routes to enable new applications of these materials. Magnetomechanical coupling was discussed in the context of ferromagnetic alloys including NiMnGa (R. O'Handley, MIT; M. Kohl, IMT Karlsruhe), and integration of these materials with ferroelectric materials was demonstrated as a means to resonance (J.R. Petrie, Cornell) and actuation power (I. Takeuchi, Univ. of Maryland) in such systems. Mechanically active liquids and organic/macromolecular solids were presented as a growing system of materials that can be tuned to absorb energy (G. McKinley, MIT), actuate through interaction with light (P. Palffy-Muhoray, Kent State; S. Yang, Univ. of Pennsylvania) or chemical potentials (P. Mather, Case Western), and control the adhesion, shape, and proliferation (multiplication) of biological cells (K.J. Van Vliet, MIT). General modeling approaches to shape memory were presented (D.C. Lagoudas, Texas A\&M; C. Janetti, Univ. of Pennsylvania; R.C. Pond, Univ. of Liverpool), and quantum-based modeling of organic active materials (D. Scherlis, MIT; A. Strachan, LANL) was discussed as a new computational approach to modeling mechanically active materials.

Symposium Support: Boston Scientific Corp.

\section{Experimentalists and Theorists Share Notes on Biological Materials (See MRS Proceedings Volume 844)}

In Symposium Y, modelers using $a b$ initio, molecular dynamics, finite element, and constitutive modeling for biosystems interacted with experimentalists using AFM, nanoindentation, optical tweezers, and various mechanical test systems to investigate the mechanical behavior of biological and bio-inspired materials. Among the participants were several researchers from the mechanics of biological systems community. In addition, talks were given by two clinical experts, K.-N. An (Mayo Clinic) and B. Snyder (Harvard Medical School), who deal with clinical aspects of mechanical behavior of biological materials at the tissue level.

Biological and bio-inspired materials often exhibit nanoscale architecture as well as structural hierarchy over many length scales. The mechanics of these systems is complex due to nanoarchitecture, the role of nanoscale interfaces, and the load transfer mechanisms at interfaces. Developing robust realistic prediction methodologies and simulations of mechanical responses

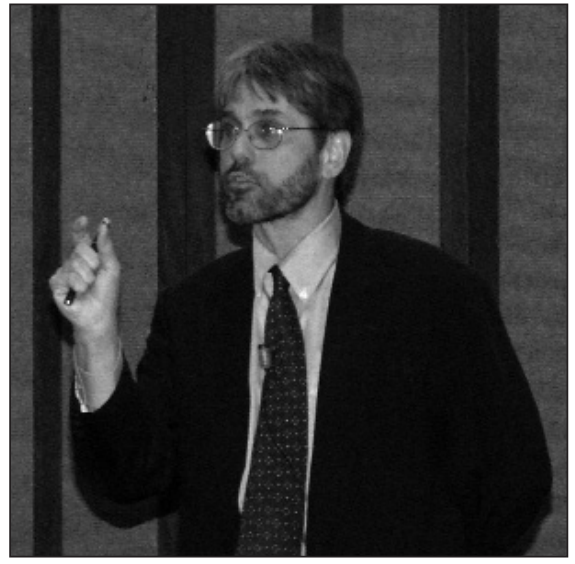

Frank S. Bates (Univ. of Minnesota) delivers the 2004 MRS David Turnbull Lecture.

in these systems requires collaboration between many disciplines of engineering and science. In a joint session with Symposium R, the use of nanoindentation for experimental measurement of mechanical response in biological materials was reported. Also, the mechanical response measured using nanoindentation at cellular, molecular, and tissue levels was reported.

D. Katti (North Dakota State) is interested in the advanced mechanical properties of nacre that are comparable to modern high-technology ceramic materials. The eventual goal of his group's research is the simulation-based design of biomimetic nanocomposites. Recently, the group addressed the role of nanoasperities in shaping the properties of nacre. Additional research focused on experimental investigations based on dogboneshaped samples of nacre, in which Katti's group studied fracture mechanisms and fracture surfaces. In addition, the researchers discovered platelet-platelet interlocks from the observation of fracture surfaces, which they believe is one of the key reasons for the strength and high toughness exhibited by nacre. Using finite element modeling simulations of nacre with interlocks undergoing loading, the scientists observed that these interlocks show progressive failure that along with deformation of the organic phase and aragonite platelets, leads to toughening. This suggests that the interlocks play an important role in the relatively large strain behavior of nacre before it fails.

M. Greene (Northwestern) presented recent results of interdisciplinary studies of the characterization of DNA properties using a clever combination of AFM, pic- ture analysis, and theoretical worm-like chain modeling. The scientists are interested in investigating the behavior of bare DNA molecules, which are normally surrounded by histone proteins in the cell nucleus. The analysis method is based on using AFM to generate a series of trajectory snapshots of the configuration of the DNA molecules. Picture analysis methods that perform contour tracking of the molecules are used to extract information about the trajectories. Greene and his colleagues are particularly interested in determining the contour length as well as how the molecules bend and deform. These data can be used to extract parameters that allow the description of the molecules such as elastic rods near equilibrium modeled as worm-like chains. Parameters for such model chains are obtained using parameter fitting to experimental results analyzed by the picture analysis methods. Based on these methods, the persistence length of the DNA molecule is found to be about $62 \mathrm{~nm}$, which is in reasonable agreement with previous research results for molecules of the length investigated. The properties of the bare DNA stand in significant contrast to the structure and properties of DNA bound to histones, underscoring the ability of proteins to deform DNA by electrostatic means. The new analysis procedure described in Greene's talk is an interesting and promising example of a multiscale hierarchical modeling scheme that enables the coupling of experimental data with theoretical modeling.

A few more highlights include two talks by S. Suresh's group (MIT) on the mechanics of red blood cells and the relationship of their response to malaria and a philosophical perspective on biomimetics presented by J. Vincent (Univ. of Bath, U.K.).

Symposium Support: Hysitron and NSF.

\section{Molecular Motors and Microlenses Inspired by Biology}

In the session on bio-optics and biophotonics in Symposium Z on Bio-Inspired and Bio-Derived Materials and Processes, J. Aizenberg (Bell Labs/Lucent) discussed biologically formed microlens arrays, focusing on brittle stars, which are lightsensitive. The skeleton of the brittle star consists of five components made of calcite, and the underlying substrate was shown to be a complete single crystal. The dorsal arm plate has unusual and very regular spherical structures, which were determined to be lenses. Quantitative characterization of the microlenses was carried out; spot size at the focal plane was $\sim 3 \mu \mathrm{m}$, with a light enhancement of $\sim 50$ at the focal point. Detailed studies revealed that the organism is able to correct for spherical 
aberrations and birefringence. At the focal point for each lens element, a bundle of nerves was observed whose size is about the same as the focal spot size. Similar to adjustable sunglasses, the brittle star uses these lenses to detect the light intensity and injects pigment to the surface, leading to a color change. Aizenberg mentioned that this organism has the only known inorganic compound eye. She also said that the calcite crystal is mechanically reinforced by intercalated proteins, which leads to unusual mechanical properties. As a multifunctional material, the lenses of the brittle star serve as an inspirational system for technology. The system, she said, is a nearly perfect combination of microlenses for "crystal-clear vision."

W.B. Sherman from New York University focused on DNA-based "biped" molecular motors that allow forward and backward motion to solve materials transport problems. The biped robot is completely made out of DNA, with flexible linker molecules connecting its "feet." Monitoring is achieved by attaching psoralen molecules, which are small molecules that can intercalate between bases. Under UV irradiation, they are found to covalently bond thymines in their neighborhood, enabling the detection of which feet are set in place (in contact) or not (no bonding). Sherman showed a schematic representation of how progression is achieved by a completely different mechanism than kinesin. The motion of the "bipeds" is much slower than that of kinesin, however; this system only took one step per hour, although it could likely go several orders of magnitude faster. Different features of how these systems are synthesized and how they can be utilized were discussed. For example, branch junctions can be built by sequence programming that specifies three, four, or more armed junctions. Possible applications of these techniques include computational systems, building nano assembly lines, as well as molecular weaving, braiding, and threading. They provide an interesting alternative to kinesin-type molecular motors.

H. Hess (Univ. of Washington, Seattle) described recent progress in using biomolecular motors as engines for nanoscale transport and assembly. The molecular motor hydrolyses ATP, leads to a conformational change that pushes the whole molecule forward, a maneuver that is repeated up to about 100 times per second. The main question of Hess's research was to uncover strategies for transporting matter at various scales. Whereas diffusion is efficient over short distances, pressure-driven fluid flow is effective over macroscopic distances. In contrast, Hess said that active transport by motor molecules such as kinesin is highly effective in the mesoscopic regime between these two extremes. The research objective of Hess's group over the last few years has focused on finding solutions to build nanoscale shuttle systems that may transport nanoscale materials and molecules over such intermediate distances. This may solve critical transport needs in nanoscale bio-inspired technologies.

Motor molecules could also be used to study the strength of receptor-ligand bonds, representing an appealing implementation of a piconewton force meter, Hess said, as he outlined several links to materials science. For instance, molecular motors on microtubules could be used to investigate mechanical properties such as fracture and elasticity as well as selfassembly driven by biomolecular motors. He also mentioned some recent studies on the lifetime of bio-nanodevices.

Symposium Support: Biomolecular Materials $\mathcal{E}$ Interfaces, DARPA, Dow Corning Corp., Genencor Intl., SNL, and the Whitaker Foundation.

\section{Micro- and Nanofabrication Applied to Medicine (See MRS Proceedings Volume 845)}

The purpose of Symposium AA was to explore the capabilities of the micro/ nanoscale in the design and fabrication of devices for applications in biology and medicine. Particular emphasis was placed on elucidating mechanisms of cell interactions with materials surfaces using a variety of micro/nanotechnology-based materials processing and characterization methods. For example, technology used originally for MEMS is now being used to create novel protocols for surface modification of biomaterials, serving a variety of applications such as the regulation of cardiac myocyte function and the creation of implantable conduits for neuronal cell transplantation (T. Desai, Boston Univ.). Several investigators used innovative processing methods for the creation of nanofiber-based scaffolds for repair of bone tissue and the spinal cord. S. Stupp (Northwestern) described a unique nanofiber scaffold based on peptide amphiphile molecules that has shown the ability to selectively differentiate neural progenitor cells to neurons and not astrocytes, cells known to complicate spinal cord injuries. Novel peptides have also been synthesized to target specific integrin receptors for the selective adhesion of cells to implant surfaces (A. Garcia, Georgia Tech). Other researchers are investigating the optimal spacing of adhe- sion peptides to optimize cell function (D. Mooney, Harvard). Adhesive peptides have also been incorporated within injectable polymeric hydrogels for applications in tissue engineering (A. Mikos, Rice). In other studies, researchers explored the fascinating effects of nanoscale topography on the adhesion and function of cells on various polymeric, ceramic, and metallic biomaterial surfaces (R. Bizios, RPI, T. Webster, Purdue). Individual nanoscale particles of biodegradable polymers have also been synthesized for localized drug delivery to combat various forms of cancer (M. Saltzman, Yale).

Symposium Support: Army Medical Research and Material Command, Charlotte Research Inst., $\mathrm{NIH}$, and the Whitaker Foundation.

\section{Polymers Combine in a Multitude of Ways}

Symposium BB on Multicomponent Polymer Systems: Phase Behavior, Dynamics, and Applications covered block copolymers, nanocomposites, associating polymers, and blends. Symposium BB also hosted the Turnbull Lecture by Frank S. Bates (Univ. of Minnesota).

Block copolymers continue to be a wide-ranging field of study. R. Register (Princeton) presented a fundamental study of crystallization and chain folding within the confined block copolymer domains, finding a stepwise increase in domain thickness with molecular weight corresponding to an integral number of folds. T. Lodge (Univ. of Minnesota) delighted the audience with a zoology of micelle types produced by strongly segregating triblock copolymers in water, including the "hamburger," "onion," and "double-yolk egg."

A series of presentations from the group of R. Composto (Univ. of Pennsylvania) detailed their extensive work on polymer-blend phase separation in thin films using a powerful combination of ion-beam depth profiling and AFM to characterize morphology and dynamics. Recently, the Composto group has initiated the study of thin-film polymer-blend dynamics with the new twist of adding nanoparticles.

Multiple presentations from the Air Force Research Laboratory (WrightPatterson) highlighted the wide range of experimental methods that provide insight into the dynamics of polymer-clay nanocomposites, including NMR and impedance spectroscopy. M. Dadmun (Univ. of Tennessee) is using H-bonding to promote clay dispersion in nanocomposites by using copolymers of polystyrene and poly(vinyl phenol). K. Winey's group (Univ. of Pennsylvania) is focusing efforts 
on improving the electrical and thermal conductivity in polymer nanocomposites and reported an eightfold increase in thermal conductivity in a nanotube/epoxy composite.

In an impromptu presentation given when scheduled speakers withdrew, C. Leibig (Dow) commented on the need to revise the curriculum in polymers to include more mechanics and on the challenges of introducing polymer-clay nanocomposites into a product. Also, R. Oliver (International Centre for Life, U.K.), having recently served on a Royal Society committee on nanotechnology, presented their findings and recommendations.

Symposium Support: Dow Chemical Co. and ICI Group (U.K.)

\section{Liquid-Crystal Elastomers and Gels Considered}

Symposium CC covered liquid-crystal (LC) elastomers and gels, numerous aspects of liquid-crystal materials, LC/polymer nano- and microstructures, and novel photonic applications. In the elastomer area, D. Broer (Philips Research Labs.) demonstrated an innovative MEMS device using LC networks. The LC networks bend when heated, and the deformation process is fully reversible. An artificial arm using the thermal expansion of LC networks is foreseeable in the near future. In the fast-response LC modulator area, A. Glushchenko (Kent State) reported a stressed LC layer with a sub-millisecond response time. Usually, the LC response time has a quadratic relationship with cell gap. In the stressed LC cell, response time is basically independent of LC thickness. Therefore, a stressed LC cell can be made with a large gap while maintaining a fast response time. Glushchenko's group has demonstrated an $800 \mu \mathrm{m}$ stressed cell. The response time is about $5 \mathrm{~ms}$, and operation voltage is only $1 \mathrm{~V}_{\mathrm{rms}} / \mu \mathrm{m}$.

In the area of novel LC materials, A.P. Alivisatos (UC-Berkeley) introduced an LC phase formed by semiconductor nanorods. Each CdSe nanorod is $4 \mathrm{~nm} \times$ $40 \mathrm{~nm}$. The rods are dissolved in a solvent. As the solvent evaporates, the rod gets denser and forms the LC phase.

In the photonics area, $\mathrm{H}$. Coles's group (Cambridge) reported lasing phenomena in a laser-pumped cholesteric LC cell configuration. The cholesteric LC is doped with a fluorescent dye. Upon laser excitation, the cholesteric cell behaves like a laser cavity. The stimulated dye's fluorescence is amplified and laser action starts. In another talk from the same group, M. Pivnenko (Cambridge) reported lasing

\section{Graduate Students Receive Gold and Silver Awards}

During the Awards Ceremony held on December 1 at the 2004 Materials Research Society Fall Meeting, graduate student finalists received Gold and Silver Awards.

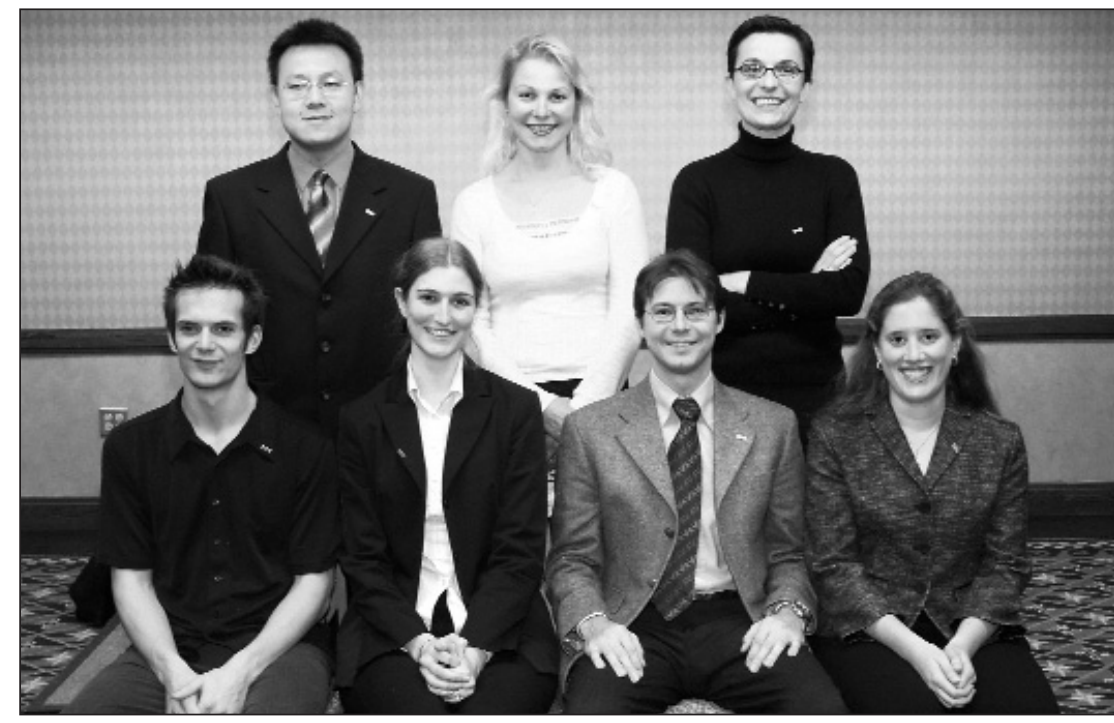

Gold Graduate Student Awards went to (front row, left to right): Andrew Armstrong (The Ohio State University), Delphine Dean (Massachusetts Institute of Technology), Stefano Piscanec (Cambridge University), and Teresa M. Barnes (Colorado School of Mines); (back row, left to right): Donghai Wang (Tulane University), Julia R. Greer (Stanford University), and Ingrid A. Rousseau (University of Connecticut). Not shown are Jifeng Liu (Massachusetts Institute of Technology) and Tao Xu (Clemson University).

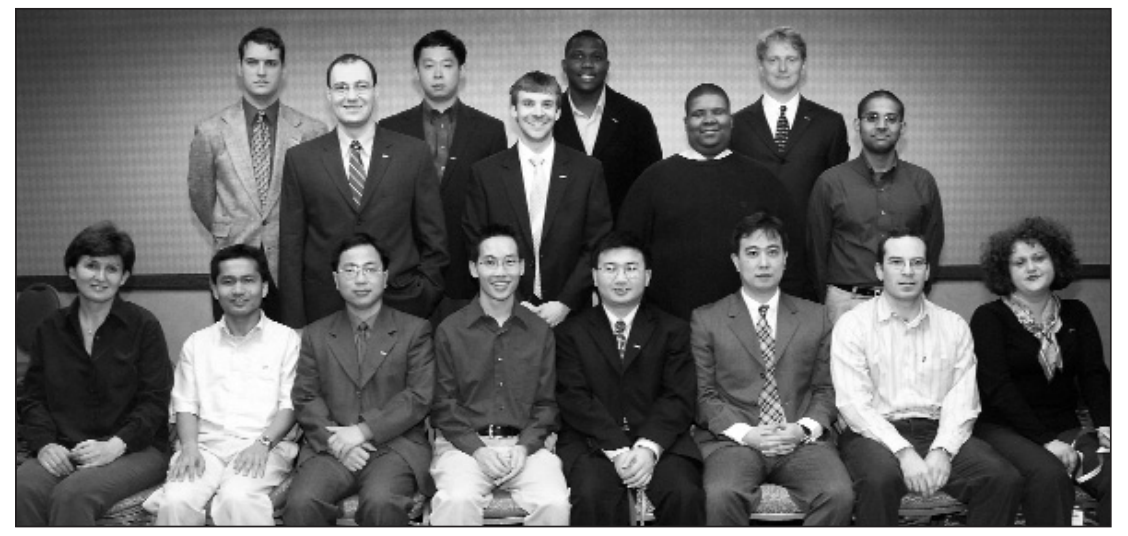

Silver Graduate Student Awards went to (front row, left to right): Svetlana $\boldsymbol{M}$. Mitrovski (University of Illinois, Urbana-Champaign), Zuruzi Abu Samah (University of California, Santa Barbara), Jingsi Yang (Rutgers, The State University of New Jersey), Homan Yuen (Stanford University), Yong Xiang (Harvard University), David Guangjun Xu (University of Illinois, UrbanaChampaign), Matthew R. Dreher (Duke University), and Doinita Neiner (University of New Orleans); (back row, left to right): Matthew H. Kane (Georgia Institute of Technology), Ion Bita (Massachusetts Institute of Technology), Jian Zhang (University of North Carolina, Chapel Hill), Brian H. Clare (University of Wisconsin, Madison), Jefferson E. Rose (University of California, Los Angeles), Trevor Hoskins (Georgia Institute of Technology), Anders Ballestad (University of British Columbia), and Ashwin Ramasubramaniam (Brown University). Not shown are Evgenia M. Kim (Moscow State University), Daniel J. Solis (Massachusetts Institute of Technology), and Wenguang Zhu (Chinese Academy of Sciences). 
behavior in a blue-phase LC. The bluephase LC is like a 3D photonic crystal. If the pumping beam is incident in the $x$ axis, the laser emissions would occur in both the $y$ and $z$ axes. M. Ozaki (Osaka Univ.) reported a tunable laser action in nanostructured LCs. Since the cholesteric pitch can be tuned by voltage, the output laser wavelength can be tuned as well.

In the ferroelectric LC area, D. Walba (Univ. of Colorado) reported a novel main-chain FLC polymer fiber. Through a skillful polling process, the FLC molecules align well inside the fiber.

\section{Symposium X Covers the Route of Materials from Innovation to Societal Impact}

How do materials develop from their birth in the laboratory to having an impact on modern society? Innovators, researchers, and entrepreneurs tackled this question in four lunchtime sessions for nonspecialists grouped as Symposium $X$, Frontiers of Materials Research. Beginning with established and novel innovations in optics, Donald Keck (SUNY-Big Flats) covered optical fibers, while Lisa Dhar (InPhase Technologies) looked to holography as a leading contender for the next generation of optical storage. Keck, one of the inventors of the first operational optical fiber for communications at Corning, said it took 16 years for the innovation to become profitable for the company. Now, over three decades after the original work, more than 800 million kilometers of installed optical fiber forms the communications network backbone, enabling the Internet and providing for society's ever more connected world. Dhar said that holographic storage, with its ability to store up to terabytes of information on a single medium and to transfer data at up to gigabits per second, can satisfy the growing demands for storage in applications ranging from high-end markets such as archiving to consumer applications such as video and multimedia distribution.

Currently, medical devices form a $\$ 50$ billion industry, with over 11,000 companies in the United States alone, said Rebecca Bergman (Medtronic Inc.). The innovations began with materials, then moved to bioactive and now biointeractive devices in the past three decades. As part of a regulated industry in which devices and not materials per se go through a long, expensive approval process, innovations in biomaterials tend to be somewhat stifled. Medtronic, for example, uses only 25 polymers and 11 metals in all of its approved devices. Looking forward, however, Bergman said that the future will see a tremendous transformation in healthcare, from treatment to cure and prevention.

Developments in bulk metallic glasses have also seen an evolution in the past few decades, with the most significant advances occurring in recent years. These include the development of $\mathrm{Cu}$ - and Fe-based amorphous alloys in large-enough sizes that they can be considered for structural applications, said William Johnson (CalTech).

Jim Daughton (NVE Corp.) described the innovations made in magnetic spin devices, beginning with giant magnetoresistance devices discovered in 1988. This launched a period of intense research into the dependence of electron conduction on their spins in numerous materials and device configurations. Daughton said that magnetoresistive random-access memory (MRAM) is very close to commercialization. MRAM has the potential advantages of high density, high speed, nonvolatility, and write-cycle durability, he said.

Reactive foil technology, in which multilayer foils react exothermically, makes up a new body of nanostructured materials that can be used to solder or braze components together. Omar Knio (JHU, Reactive Nanotechnologies) said that the use of reactive foils as a local heat source eliminates the need for torches, furnaces, or lasers; speeds up the soldering and brazing processes; and dramatically reduces the total heat that is needed.

Looking toward the future, innovations in nanotechnology may meet future needs in energy and security, as speculated by Richard E. Smalley (Rice) and Malcolm R. O'Neill (Lockheed Martin Corp.). Smalley, the 1996 Nobel Laureate in chemistry, said that the overriding societal problem in the next 50 years will be meeting global energy needs and that nanotechnology can play a critical role in the solution. Likewise, in the area of defense, $\mathrm{O}^{\prime} \mathrm{Neill}$ listed the role that nanomaterials can play in aerospace systems to create lighter-weight and stronger materials and smaller components than are currently available, advanced material functionalities, composites, and materials/structures to enable "single stage to orbit" flights. For all of these needs, he said, "nano" is becoming a definitive technology.

In the photonic LC area, J. Martz (EPFL, Switzerland) reported LC-infiltrated 2D photonic crystals. The $2 \mathrm{D}$ photonic crystal is made of $\mathrm{GaAs}$ waveguides. The length of the waveguide is about $1 \mu \mathrm{m}$. A surfactant was used as a monolayer for aligning the E7 LC mixture. The bandgap tuning is achieved by the thermal-induced LC refractive index change. F. Du (Univ. of Central Florida) used an external electric field to tune the properties of a LC-infiltrated photonic-crystal fiber. The fiber consists of an air core surrounded by an array of air holes in silica so that light-guiding is achieved by the photonic bandgap effect. By inserting liquid crystal into the fiber core and cladding the air holes, Du's group demonstrated an electrically tunable fiber optical switch with over $30 \mathrm{~dB}$ attenuation at $60 \mathrm{~V}_{\mathrm{rms}}$ for a He-Ne laser beam. This LCfilled photonic-crystal fiber could find useful applications in fiber-optic communications systems.

Symposium Support: AFRL.

\section{Organics and Nanocomposites Establish Optical Niche} (See MRS Proceedings Volume 846)

Symposium DD, Organic and Nanocomposite Optical Materials, focused on the theory, synthesis, and characterization of organic and nanocomposite materials. In addition, the symposium was organized to encourage interactions between researchers from chemistry, chemical engineering, materials science, and engineering. While the symposium provided a broad range of information on nanocomposite optical materials, there was a significant focus on nonlinear optics, OLEDs, and photonic-bandgap structures.

The enhancement of the nonlinear optical properties of organic photonic-bandgap structures was the focus of early presentations. Phase-matching for third-harmonic generation from IR to visible was shown to be possible near the band edge of these photonic crystals (P.N. Prasad, SUNYBuffalo). In addition, modeling and simulation of nonlinear optical processes (R. Pachter, AFRL) and nonlinear pulse propagation (H. Agren, Royal Inst. of Tech., Sweden) were presented. Talks focusing on the synthesis of new molecules with enhanced nonlinear optical properties were also presented.

Subsequent sessions focused on the linear optical properties of polymers and nanoparticles relevant to OLEDs. A number of talks captured recent exciting developments in electroluminescence from quantum dots embedded within OLEDs. J. Steckel (MIT) presented efforts on blue-emitters and S. Coe-Sullivan (MIT) covered the optimization of effi- 
ciency and the achievement of saturatedcolor red, green, and blue using these hybrid devices. In addition, efforts focused on blue light-emitting polymers (A. Holmes, Cambridge), and widebandgap host materials for blue-emitters (L. Sapochak, PNNL) were presented.

The final talks centered on the fabrication and characterization of photonic-bandgap structures. These included presentations on photonic crystals fabricated using holographic interferometry (C. Ullal, I. Bita, E. Thomas, MIT), atomic layer deposition (J. King, Georgia Tech), and electronbeam lithography (G. Subramania, SNL). It was demonstrated that it is possible to fabricate uniform, periodic, organic-based photonic-bandgap structures with predictable optical properties.

Symposium Support: ARL.

\section{Hybrids Expand Functionality (See MRS Proceedings Volume 847)}

The tone for Symposium EE on Organic/Inorganic Hybrid Materials was established by E. Duget of the University of Science and Technology of Bordeaux, who introduced the concept of "Janus," or two-headed, hybrid particles, each side functionalized in a completely different manner. Thereafter, a number of researchers discussed the use of templates to produce mesoporous inorganic structures that could be further functionalized with organic moieties. This group included R. Anwander (Univ. Munich), who described novel ways of making mesoporous silicate structures and functionalizing their surfaces. C.J. Brinker (Univ. of New Mexico) highlighted work that included capturing living cell structures within sol-gel-created inorganic structures.

In the arena of self-assembly, S. Stupp (Northwestern) created organic structures that mimic surfaces that induce the crystallization of materials ranging from hydroxyapatite, the primary constituent in bone, to semiconductor structures based on CdS. S. Glotzer (Univ. of Michigan) described theoretical modeling tools that enable the identification of 3D structures that can evolve as a result of variations in specific topological relationships between covalently bonded organic-inorganic building blocks that self-assemble.

In the area of sophisticated analysis of topological and structural features in hybrid materials, F. Babonneau, C. Gervais, and C. Bonhomme of Paris VI described different aspects in the use of solid-state NMR to analyze both organic and inorganic components in hybrid materials. M. Linden (Univ. Turku, Finland) described the use of in situ SAXS/XRD to examine the formation of mesoscopically ordered materials.
A. Usuki (Toyota) led a series of talks describing recent work on organically modified clays with a discussion on polyolefin clay hybrids. L. Drzal (Michigan State Univ.) described complementary work on the exfoliation of graphite to make novel organically modified graphite sheet nanocomposites. U. Wiesner (Cornell) described some novel phases generated during explorations of multifunctional nanostructured hybrids.

K. Matsukawa (Osaka Municipal Technical Research Inst.) led off a series of talks on hybrid materials used for electronic and photonic applications with a talk on patterning substrates with photopolymerization/bleaching of polysilanes on selected substrate materials. D. Loy (LANL) discussed alkylene-bridged polysilsesquioxanes for the formation of porous xerogels with potential low- $\kappa$ dielectric applications. J. Kieffer (Univ. of Michigan) described modeling of silsesquioxane hybrid materials packing, selfassembly, and methods of estimating physical properties.

A. Sellinger (IMRE, Singapore) discussed the use of polyphosphazene rings as novel cores for the creation of new OLED materials with exceptional luminescent properties. In the same arena, H. Mutin (Univ. Montpellier) described the synthesis and characterization of phosphorus-containing hybrid materials.

The use of hybrid membrane materials with properties of value as fuel cell membranes (e.g., by P. Gomez-Romero, Univ. Barcelona) and for separation applications (e.g., by F. Faluja, Univ. Montpellier) were also covered.

Symposium Support: Accelrys and AFRL.

\section{Inorganics Range from Single- Crystalline to Nanocrystalline}

\section{(See MRS Proceedings Volume 848)}

The synthesis of new materials in the area of solid-state chemistry of inorganic materials (Symposium FF) continues at a heady pace. On the one extreme, the controlled synthesis of nanomaterials is more reliable, with Y. Xia (Univ. of Washington) showing the influence of polymer capping units on forming uniform metallic nanowires, while both S. Brock (Wayne State Univ.) and I. Shweky (Hebrew Univ.) demonstrated the use of myristic acid to control the growth of nanorods and particles of metal pnictides. To the other end, large single crystals are needed for certain practical applications. J. Kolis (Clemson) showed the hydrothermal synthesis of borates for deep-UV applications; P. Guptasarma (Univ. of Wiscon$\sin$ ), the floating zone growth of superconducting $\mathrm{Bi}_{2} \mathrm{Sr}_{2} \mathrm{CaCu}_{2} \mathrm{Oy}$ crystals, and
W. Schnick (Univ. Munich), the growth of nitrides and oxynitrides with luminescent properties.

The synthesis of framework structures is proving to be a robust field. P. Lightfoot (Univ. of St. Andrews) focused on the crystal chemistry of metal fluorides, while P. Feng (UC-Riverside) illustrated the diverse chemistry in chalcogenide frameworks and Z. Ni (Univ. of Michigan) showed the remarkably stable metalorganic systems. These open frameworks may prove useful in gas separation or storage, catalysis, and luminescent applications. J. Badding (PSU) went to the opposite extreme, using commercial photonic fibers as templates for depositing composite structures with unique photonic and electronic behaviors.

Some highly functional materials were presented. M. Barsoum (Drexel) and J. Spanier (Drexel) highlighted carbides and nitrides, such as $\mathrm{Ti}_{3} \mathrm{SiC}_{2}$, which combine many of the best properties of metals and ceramics (ductility, machinability, creep resistance, and oxidation resistance). D. Mitzi (IBM) explained that high-mobility metal chalcogenide films may find use in flexible electronics. J. Chan (Louisiana State) discovered new magnetic intermetallics, while R. Seshadri (UCSB) explained induced magnetism in widebandgap host materials such as $\mathrm{ZnGa}_{2} \mathrm{O}_{4}$.

J.C. Schoen (MPI) utilized ergodic theory on the energy landscape to predict structural stability of as-yet unsynthesized materials. S. Jobic (CNRS) dissected refractive indices, pointing toward optimizing optical materials. N. Spaldin (UCSB) turned attention to ferromagnetic materials, such as doped $\mathrm{ZnO}$ and $\mathrm{TiO}_{2}$, for spintronic applications.

Symposium Support: ACS/Petroleum Research Fund, NSF, and Solid State Chemistry.

\section{New Directions Explored for Creating Mesoscale Materials from}

Nanostructured Building Blocks

Symposium GG, Mesoscale Architectures from Nanounits-Assembly, Fabrication, and Properties, contained a diverse array of presentations ranging from the theory of nanoscale materials to new directions for the biologically enabled synthesis of nanostructured materials. An early highlight was a special session with Symposium C on Emerging Paradigms in Nanopatterning and Mesoassembly in which A.P. Alivisatos (UC-Berkeley), R. Dammel (AZ Electronic Materials), G. Whitesides (Harvard), C. Mirkin (Northwestern), and S. Chou (Princeton) shared their viewpoints on the future of lithography and alternative "bottom-up" assembly routes for creating large-scale functional structures from nanoscale building blocks. 
F. Stellacci (MIT) covered phaseseparated ligand shells in metal nanoparticles. The capping ligands on gold nanoparticles can be used to tune many of their properties, including solubility and electronic and optical properties. It is known that mixtures of ligands (e.g., hydrophilic and hydrophobic) on flat gold surfaces show random phase segregation. Stellacci found, however, that on gold nanoparticles, the phase separation is ordered and regular with well-defined banded hydrophobic and hydrophilic domains. He found that this behavior has nothing to do with the crystal core, but rather is due to the topological curvature of the nanoparticles. Stellacci said this phenomenon can be explained by the "hairy ball theorem," which states that vectorial order cannot propagate on a sphere until there are two defects at opposite poles. The ligands at the poles are readily amenable to exchange, and this can be used to string together nanoparticles in chains, generating a "nano-nylon." Mixtures of ligands can be used to carefully tune the width of the hydrophilic and hydrophobic bands. A demonstrated application of these novel materials has been the achievement of greatly reduced nonspecific protein adsorption on nanoparticle surfaces, which could significantly enhance the biocompatibility of gold nanoparticles.

K. Kern (MPI, Stuttgart) focused on the directed assembly of metalorganic architectures through non-covalent interactions. The tobacco mosaic virus has an especially attractive cylindrical structure with a length of $300 \mathrm{~nm}$ and an inner channel that is $2-4 \mathrm{~nm}$ in diameter. Kern used these viruses as templates for the growth of metal nanowires. The oxo ligands at the outer surface of the virus and the amine ligands lining the channel provide an opportunity for engineering selective metal-ligand interactions to target either the surfaces or the inner channels for metallization. Since these viruses can be genetically engineered to introduce different ligands at different sites, they can provide a versatile route toward different nanoscale architectures. Another approach developed by Kern involves the use of molecular linkers with functional groups that coordinate to metal atoms to form unique hybrid metalorganic architectures on surfaces. Careful choice of ligands allows the separation between metal atoms to be controlled with great precision. These hybrid architectures thus have three components: a metal center with potential magnetic or catalytic activity, an organic molecular component with recognition properties, and an empty cavity with possible use in adsorption or as a nanoreactor.

Novel thermal, electrochemical, optical, magnetic, and mechanical properties, and possible applications of macroscale assemblies in sensing, actuation, switching, and information storage, were described by Y. Lu (Tulane), R. Baughman (Univ. Texas, Dallas), P. Ajayan (RPI), and L. Allen (UIUC). The theoretical aspects of assembly and predicting properties of nanostructured assemblies were described by H. Terrones (IPICyT, Mexico) and others.

Symposium Support: ARO and Mexican Society of Nanoscience and Nanotechnology.

\section{Carbon Nanotubes Show Extraordinary Properties, although Isolation is Elusive}

Symposium $\mathrm{HH}$ brought together physicists, chemists, material scientists, and engineers working in the field of carbon nanotube research. The various extraordinary properties of carbon nanotubes, such as ballistic electron transport and their extremely high Young's modulus, give rise to manifold research subjects. The symposium covered the main aspects of carbon nanotube research, from synthesis and processing through characterization and applications.

In the area of synthesis and processing, proper ways to produce or select specific nanotubes with desired properties are still lacking. A special CVD method for achieving control in localization, size, and orientation using nanotemplates prepared from amphiphilic diblock copolymers was presented by J. Lu (Univ. of Toronto). A further remaining task relevant for applications is the separation of semiconducting from metallic nanotubes, which was addressed by several speakers.

The importance of Raman spectroscopy for the characterization of carbon nanotubes was reviewed by $\mathrm{Ch}$. Thomsen (Technical Univ., Berlin). Thomsen and S. Piscanec (Cambridge) pointed out that contrary to general opinion, a Raman feature that so far has been attributed to Fano resonances in metallic nanotubes should be explained in another way. M. Dresselhaus (MIT) analyzed less pronounced features of Raman spectra that so far only were discussed less intensively.

Using TEM, J.C. Meyer (MPI, Stuttgart) was able to visualize the same individual carbon nanotubes that had been investigated before by different physical methods such as Raman spectroscopy or electrical transport measurements. N. de Jonge (Philips, Eindhoven) replaced standard TEM electron sources by single
MWNTs, which improves the image quality because the electron energy distribution becomes much narrower.

B.J. Hinds (Univ. of Kentucky) selectively functionalized carbon nanotube ends by side-wall protection in aligned membrane structures. Prominent applications of carbon nanotubes such as sensors (J.-C.P. Gabriel, Nanomix), active and passive electrical components within siliconbased microelectronics (G.S. Duesberg, Infineon Technologies), and as AFM tips (J.D. Schumacher, Univ. of South Florida, Tampa) were discussed.

Symposium Support: Applied NanoFluorescence, Foster-Miller, NanoTechLabs, Wake Forest Univ. Graduate School of Arts and Sciences, and Wake Forest Ctr. for Nanotechnology and Molecular Materials

\section{Low-Dimensional Systems Studied}

Symposium II, Modeling of One- and Two-Dimensional Materials, explored computational applications and theoretical developments as they relate to the design, synthesis, properties, and understanding of low-dimensional nanomaterial structures and assemblies. The first session centered on electron transport in 1D systems, opened by M. Ratner (Northwestern). Ratner said that molecular transport junctions comprise effectively linear structures coupled to electrodes at either end. Using what has become standard nonequilibrium Green's function approaches, Ratner analyzed molecular substituent effects and, in particular, inelastic effects on junction transport. Nano-scalable behavior, under extreme confinement, was described by U. Landman (Georgia Tech).

A number of topics were covered, including the generation, stability, and breakup of nanostructures; the emergence of magnetism in free and surfacesupported small palladium clusters; gold nanocatalysis; and the rheological and nanotribological properties of lubricating fluids in highly confined environments. For example, Landman and colleagues have compared experiment to molecular dynamics simulations, thus gaining insight and a fundamental understanding of the most basic equation of friction. G. Hummer $(\mathrm{NIH})$ reported that carbon nanotubes offer unique possibilities to probe the properties of confined fluids and to study the transport of molecules and ions through narrow molecular channels. Simulations of the flow of water, protons, ions, and biopolymers through individual nanotubes and nanotube membranes highlight key elements of different molecular translocation mechanisms. For example, Hummer said that proton diffusivity is higher along one-dimensionally 
ordered water chains in small-diameter carbon nanotubes than in bulk water; most recently, the interaction of water chains with homogeneous electric fields for finite closed and infinite periodically replicated tubes was also studied.

G. Schatz (Northwestern) described studies of fracture in defected carbon nanotubes and in doped and undoped ultrananocrystalline diamond films. For example, applying density functional theory and semi-empirical methods in combination with molecular mechanics calculations that use a Tersoff-Brenner potential to explore the role of vacancy defects in the fracture of carbon nanotubes under axial tension, Schatz's group found that oneand two-atom vacancy defects reduce failure stresses by as much as $\sim 26 \%$ and markedly reduced failure strains. Large holes-such as might be introduced by oxidative purification processes-greatly reduced strength, and this provided an explanation for the extant theoreticalexperimental discrepancies.

A. Balazs (Univ. of Pittsburgh) explained the problem of crack formation in composites where thin brittle films lie in contact with deformable polymer layers. Using computer simulations, Balazs showed that the addition of nanoparticles to the polymers yields materials in which the particles become localized at nanoscale cracks and effectively form patches to repair the damaged regions. Surfaces that contain a nanoscopic notch were described; molecular dynamics simulations were used to determine the particle and polymer distribution on the damaged surface. The calculations revealed that a high volume fraction of relatively large particles was localized in the notch. The polymer melt induced a depletion attraction between the particles and surface and thereby drove the nanoparticles into the defect. The morphology obtained from the molecular dynamics simulation served as the input to the lattice spring model, which enabled the determination of the mechanical properties of the nanocomposite-coated surface. Stress concentration at the notch tip is significantly reduced by the presence of the nanoparticles. The application of such nanocomposite coatings could potentially yield defect-free surfaces that exhibit enhanced mechanical properties.

In general, computational soft nanotechnology lives in a multidimensional twilight zone, said H. Fraaije (Leiden Univ., the Netherlands). As Fraaije asserted, it is important to develop realistic computational methods for a variety of nanoscopic surface modifications, where the materials of choice are mixed formu- lations of flexible block copolymers, surfactants, and colloids. In particular, Fraaije discussed the theoretical microphase diagram of soft surface layers.

Symposium Support: Accelrys, AFRL, Asian Office of Aerospace RED, the European Office of Aerospace RED, Rice University Center for Nanoscale Science \& Technology-CNST, and the Wright Brothers Inst.

\section{Evolution of Surface and Interface Morphology Modeled}

In Symposium JJ on Modeling of Morphological Evolution at Surfaces and Interfaces, it was shown that heteroepitaxial growth methods for spontaneously organizing quantum dot and wire arrays continue to receive widespread attention for nanotechnology applications. Joint sessions with Symposia KK and U highlighted developments in the understanding and control of such self-assembly mechanisms. J. Tersoff (IBM) introduced a model for kinetically controlled critical wetting-layer thicknesses in the StranskiKrastanov growth mode. K. Kern (MPI, Stuttgart) showed STM data establishing size-induced pyramid-to-dome shape transitions in InAs growth on GaAs. M. Lagally (Univ. of Wisconsin, Madison) discussed directed self-assembly of quantum dots, employing pre-patterned substrates and SOI growth technology. Z. Suo (Harvard) described strategies for patterning and inducing the motion of islands of adsorbed molecules using electric fields.

Predictive models of UHV nanostructure growth require a detailed knowledge of mass transport mechanisms and thermodynamic forces driving compositional and morphological evolution. E. Williams (Univ. of Maryland) reviewed developments in experiments on fluctuations at steps and domain boundaries, and methods for extracting model parameters from statistical-mechanical analyses. Applications of state-of-the-art electronic structure calculations of surface structures and energetics were described by K.N. Varga (ORNL). V. Ozolins (UCLA) discussed a first-principles framework for modeling the thermodynamics underlying compositional patterning in heteroepitaxial growth of alloy layers, as well as coarse-grained kinetic Monte Carlo (KMC) modeling. Efficient hybrid KMC algorithms for film growth were discussed by L. Sander (Univ. of Michigan). R. Caflisch (UCLA)

\section{Materials Issues in Solid Free-Forming}

Symposium LL brought together discussions addressing important issues related to free-forming and other parallel processing methods for advanced materials. The symposium opened with a special address titled "Electrospraying Wings of Molecular Elephants" by John Fenn, 2002 Nobel Laureate in chemistry. The presentation elucidated the electrospray technique and the significant advantage in the use for weighting large biomolecules. Several sessions followed, with invited papers from a host of eminent scientists from around the world. The first session covered the broad field of solid free-forming, with M. Edirisinghe (Queen Mary, Univ. of London), B. Derby (Univ. of Manchester), A. Safari (Rutgers), and L. Iuliano (Univ. Modena, Italy) presenting talks on jet-based and other advanced materials-forming methods at both the nano- and micrometer scales. The following session covered 3D fabrication and applications (J. Beaman, Univ. of Texas; E. Sachs, MIT; Y. Gogotsi et al., Drexel). The second day started with a session on processing and fabrication of advanced materials (G. Babini and L. Settineri, Politecnico di Torino, Italy). The final session addressed electrohydrodynamic atomization and applications (J. De la Mora, Yale; K.-L. Choy, Univ. of Nottingham; M. Brenner, Harvard, and I. Loscertales, Univ. Malaga).

Symposium Support: ARL and Queen Mary, Univ. of London.

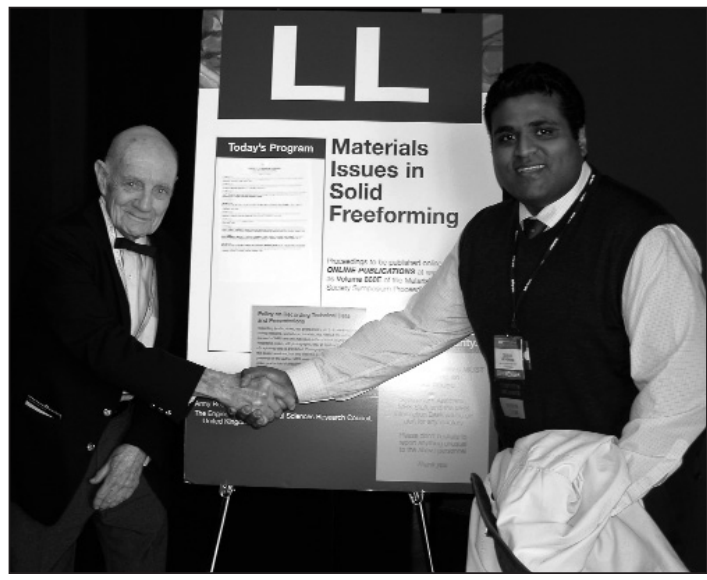

Nobel Laureate John Fenn (left) with Suwan Jayasinghe, lead symposium organizer. 
discussed a 2D continuum island dynamics approach, retaining discrete vertical layers, which can treat island growth shapes. Other contributed talks focused on 3D continuum formulations for morphological evolution.

Postdeposition diffusion and sintering of 2D clusters in metal homoepitaxial systems has been the focus of many recent studies. The corresponding behavior of 3D supported metal clusters often underlies catalyst degradation. D.-J. Liu (Ames) described how such behavior for 2D nanoscale clusters when driven by periphery diffusion deviates from classic continuum theory prediction. Atomistic details become important. T. Rahman (Kansas State) showed that many-atom processes can play a key role in cluster diffusion and that smart-KMC algorithms can search for and incorporate such processes.

Substantial progress has been realized in the modeling of crystal growth from the melt and solution, and in electrochemical systems. J. Morris (ORNL) and J. Hoyt (SNL) summarized advances in MD approaches for investigating the thermodynamic and kinetic properties of crystal-melt interfaces. L. Granasy (Budapest Univ.) and J. Warren (NIST) described phase-field modeling of the nucleation and growth of polycrystals in metals and polymers. New MD methods for computing grain-boundary mobilities and stiffnesses, underlying the evolution of grain microstructures, were described by D. Srolovitz (Princeton). Models for nanoporosity formation in dealloying, derived from KMC and continuum phase-field methods, were described by J. Erlebacher (JHU). L. Zepeda-Ruiz (LLNL) discussed KMC studies of the influence of impurities in step-mediated growth of KDP crystals. P. Vekilov (Univ. of Houston) reviewed his research detailing the role of water structuring in the crystallization of proteins from solution.

This symposium was dedicated by the organizers to the memory of Maria C. Bartelt (1962-2003), Scientific Leader for Computational Materials Sciences at LLNL. Dr. Bartelt was lead organizer for the preceding Fall 2001 MRS Symposium T. Dr. Bartelt was internationally recognized for her fundamental contributions to thin-film and materials science. She was a person of deep principles, profound determination, and unbounded optimism. She cared deeply for her friends, colleagues, and group members, and is greatly missed.

Symposium Support: Inst. of Physical Research and Technology, Iowa State Univ., LLNL, and ORNL.

\section{Nanostructuring Guided by Kinetic Barriers \\ (See MRS Proceedings Volume 849)}

Symposium KK, Kinetics-Driven Nanopatterning on Surfaces, provided a platform for the exchange of research and development advances in nanostructuring due to kinetic processes. Coupling between kinetics and mechanics, and complementation between experiments and modeling, were emphasized through a joint session with Symposia U and JJ. Fundamental issues of self-assembly, quantum dot formation, and step interactions were discussed in this session, both in modeling and in experimental work. M. Lagally (Univ. Wisconsin, Madison) presented not only patterned quantum dots, but also carbon nanotubes selectively grown on top of these quantum dots.

A fascinating array of nanostructuresfrom quantum dots to quantum fortresses-was shown on the second day. Using surfactants to modify surface kinetic barriers, such as 3D Ehrlich-Schwoebel barriers, M. Altman (UST, Hong Kong) presented a mechanism for controlling islands of Ag. The addition of a small amount of In inverts the shape of $\mathrm{Ag}$ islands.

A number of talks were presented on the use of ion beams to induce self-organization on metal, insulator, and semiconductor surfaces. Highly regular 2D arrays of $\mathrm{Si}$ clusters produced by near-normal ion bombardment were shown by B. Ziberi (Leibniz Institute for Surface Modification). Other work on the mechanisms of ripple formation showed that there is still a lot to be understood in how sputterinduced patterns form and grow. Other uses of ion beams included precise control of surface morphology and thin-film microstructure, presented by J.E. Greene (UIUC). Toh-Ming Lu (RPI), a 2004 MRS Medalist, presented novel morphologies of nanorods and nanosprings, by combining the effects of kinetic barriers and geometrical shadowing.

Symposium Support: Blake Ind.

\section{Researchers Say "Less is More" in Area of Ultrafast Lasers (See MRS Proceedings Volume 850)}

Sub-picosecond laser pulses deposit energy in non-metals by creating an abundance of free electrons by avalanche or multiphoton ionization, exciting the electrons to form a plasma, absorbing further energy into the plasma or ultimately reflecting it, and transferring energy into the solid lattice as heat. Although plasma effects place an upper limit on the useful energy per pulse, reducing pulse length well into the femtosecond range expands the window of viable processes, since many depend on intensity rather than energy. High pulse energy can ablate a significant material volume by phase explosion, yielding particles as well as atoms, in contrast to low pulse energy, said X. Xu (Purdue) in Symposium MM. Lower pulse energy is within reach, using much simpler hardware than the multistage amplified systems used for comparably intense picosecond pulses. Furthermore, the higher repetition rates available at lower pulse energy offer a way to compensate for the reduced amount of material transformed per pulse. The practical applications of ultrafast laser processing are becoming easier to envision. An ultrafast laser user facility nearing completion at Jefferson Laboratory in Virginia will expand opportunities for experimenters, said G.R. Neil. G. Williams, also of Jefferson Laboratory, said that for the first time, terahertz radiation is available with substantial power.

Ultrafast lasers enable important applications. Photosensitive glass can be activated precisely to fabricate $3 \mathrm{D}$ microdevices including fluidics, optics, and freestanding objects (K. Sugioka, RIKEN; B.N. Chichkov, LZH). Sm-containing glass can be written by a femtosecond laser and reversed by $\mathrm{Ar}^{+}$for information storage (K. Hirao, Kyoto Univ.). Only the intensity peak at the center of the beam spot can drive multiphoton processes, affording copper features smaller than the beam size (J. Fourkas, Boston College). Structural transformation can enhance the strength of glass by as much as $50 \%$ (Hirao) or can be used to write microgratings (K. Kawamura, Tokyo Univ.). Femtosecond ablation can machine materials that would otherwise ignite (Y. Picard, Univ. of Michigan). It can also provide a means of $\mathrm{ZnO}$ nanowire deposition (S. Mao, LBNL). Ultrafast ablation of organics in aqueous solution yields tailored particles as small as $3.5 \mathrm{~nm}$, which can be adapted for biosensors (M. Munier, Montreal Univ.).

Symposium Support: AFOSR, Panasonic Boston Lab., and Thomas Jefferson National Accelerator Facility.

\section{Extreme Conditions of Space Create Materials Challenges}

\section{(See MRS Proceedings Volume 851)}

Symposium NN on Materials for Space Applications offered a forum for scientific and technical discussions and analysis focused on the modifications induced by the extreme conditions of the space environment, such as ionizing-particle cocktails (containing accelerated ions, electrons, and protons), extreme temperatures, atomic oxygen, and electromagnetic radiation on materials with potential space applications. 
Many contributions were aimed at polymers and composites based on polymeric matrices. This trend reflects the need for lightweight materials with potential multifunctional, self-healing, or smart capabilities. The reduced density, good mechanical

\section{Color from Structure Brings Art to Light (See MRS Proceedings Volume 852)}

During Symposium OO on Materials Issues in Art and Archaeology, I.C. Freestone of the British Museum reported on the use of Sr isotopes to differentiate the introduction of calcium carbonate, as opposed to lime-containing sands, into ancient glass compositions. A. Sheridan and A. Shortland from Oxford University, in an analysis of Bronze Age European faience, showed the westward spread of the technology from Southwest Asia with indigenous adaptations, especially in Ireland and Britain. L. Ellis and R. Newman of the Boston Museum of Fine Arts reported a Sudanese, not Egyptian, glaze technology that was used on monumental quartzite sculptures and as a coating on smaller objects. Using Raman data, P. Colomban (CNRS) discussed the redox role of $\mathrm{SnO}_{2}$ and other components in the formation of luster glazes. J. Verbeeck (Univ. Antwerp) used STEM techniques to demonstrate that $\mathrm{Cu}$ and $\mathrm{Ag}$ nanoparticles diffuse separately into lead glazes to produce metallic luster glazes of differing colors. B. Berrie of the National Gallery of Art identified colors produced by glass inclusions and mixtures of glasses in 16th-century Venetian paintings. L. Dussubieux of the Smithsonian Institution, using ICP-MS, was able to detect trace amounts of specific mordants used in ancient textiles in comparison with Schweppe color standards. C. McGlinchey reported new mounting methods and materials used at the Museum of Modern Art for resin-coated photographs. J. Mass of the Winterthur Museum announced the development of a new technique for nondestructive, compositional depth profiling of paintings using confocal x-ray fluorescence microscopy at the Cornell High Energy Synchrotron Source. S. Miller (NASA Glenn Research Center) gave near-miraculous examples of the use of atomic oxygen treatment to clean hydrocarbon accretions from painting surfaces.

A. Murray, E. Hagen, and R. Ploeger of Queens University and D. Scalarone of the Tate Gallery presented experiments on the stability, aging, and cleaning of modern artists' acrylic emulsion paints-paints that are causing major problems for museums. M. Mecklenberg and C. Tumosa, both of the Smithsonian Institution, reported the results of a multiyear program of testing mechanical properties of aging oil paints. A. King (Purdue) used low-temperature diffusion data from historical iron objects as a predictor of material longevity. P. Vandiver (Univ. of Arizona) gave examples of craft knowledge and technologies that may be eligible for the new UNESCO Intangible Cultural Property status through a program that is similar to World Heritage Site designation. I. Ferguson (Univ. of Manchester) reported the development of new alloys and processing for the production of mokume gane (Japanese wood-grained metal).

One afternoon, the participants visited two conservation science laboratories in the Boston area: the Strauss Center for Conservation at Harvard, and the Boston Museum of Fine Arts. On Friday, they participated in a workshop, "Color from Structure," at the MIT Department of Materials Science and Engineering, at which they were able to observe Peter Houk and Fritz Dreisbach making glass murrini and paperweights embedded with the MRS logo, neriage (colored clay), ceramics with P. Vandiver, and mokume gane and other Japanese alloying and patinating methods with Deborah Krupenia.

Symposium Support: Renishaw and Rigaku MSC.

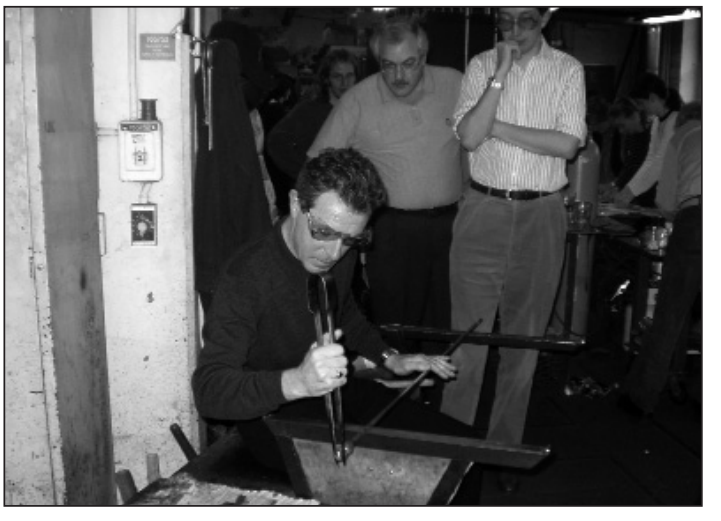

Peter Houk of the MIT Glass Lab begins to build a millefiori cane (or multicolored rod) as observed by Andrew Shortland (Oxford) and others during a workshop held in the Materials Science and Engineering Department at MIT. properties, and high thermal stability of new and emerging polymers show their potential for future space applications. Many presentations focused on polymers and composite materials obtained by dispersing various fillers within polymeric materials. Particular attention was paid to carbon nanotubes and composites obtained by dispersing carbon nanotubes within various polymers. B. Hsiao (SUNY-Stony Brook) presented the recent advances in super tough polyethylene carbon nanofibers composites, and R. Wilkins (NASA Center for Appl. Rad. Research, Texas) discussed the effect of ionizing radiation on composites obtained by dispersing carbon nanotubes within various polymeric matrices.

The latest advances in multifunctional and self-healing composites were presented by K. Wooley (Washington Univ., St. Louis) and S. Cheng (Akron Univ.), suggesting new research directions. Recent developments in materials science, including ceramics, magnetic, and conducting materials and composites with potential space applications, were reported by M. Sorescu (Duquesne Univ.) and N. Ramachandran (BAE Systems Analytical Solutions Inc.; NASA Marshall Space Flight Center, Huntsville). New emerging nanomaterials and their expected applications in space were presented by C. Grovenor (Oxford Univ.).

A distinct number of contributions focused on the effect of actual and simulated space environments on materials. The effect of ionizing radiation (protons, accelerated ions, electrons, and gamma irradiation) and electromagnetic radiation (mainly IR and UV) on polymers and composite materials was discussed by B. Briskman (Karpov Inst., Russia). The stability of polymeric materials under atomic oxygen attack —of importance for low-Earth orbits and geostationary orbits-was dissected in depth by J. Kleiman (Integrity Testing Lab. Inc., Canada) and B. Banks (NASA). The role of materials in future space projects, such as the solar sail (an innovative passive propulsion project that exploits the momentum transferred by solar photons to a large, highly reflective, low-mass structure) and the space elevator, as well as in near-term missions (to the moon and Mars) was addressed. It was concluded that future space missions depend on new materials with improved physical properties. The solar sail project requires ultrathin and light mirrors, the space elevator is based on the availability of ultratough ropes, and future missions to other planets involve materials capable of withstanding harsh environments. 